December 1999

KEK-TH-667

hep-th/9912254

\title{
Witten's Open String Field Theory in Constant $B$-Field Background
}

\author{
Fuminiko Sugino也 \\ Institute of Particle and Nuclear Studies, \\ High Energy Accelerator Research Organization (KEK), \\ Tsukuba, Ibaraki 305-0801, Japan
}

\begin{abstract}
In this paper we consider Witten's bosonic open string field theory in the presence of a constant background of the second-rank antisymmetric tensor field $B_{i j}$. We extend the operator formulation of Gross and Jevicki in this situation and construct the overlap vertices explicitly. As a result we find a noncommutative structure of the Moyal type only in the zero-mode sector, which is consistent with the result of the correlation functions among vertex operators in the world sheet formulation. Furthermore we find out a certain unitary transformation of the string field which absorbs the Moyal type noncommutative structure. It can be regarded as a microscopic origin of the transformation between the gauge fields in commutative and noncommutative gauge theories discussed by Seiberg and Witten.
\end{abstract}

\footnotetext{
${ }^{1}$ E-mail address: sugino@post.kek.jp
} 


\section{Introduction}

Noncommutative nature of space-time has often appeared in nonperturbative aspects of string theory. It has been used in a formulation of interacting open string field theory by Witten [1, 2]. He has written a classical action of open string field theory in terms of noncommutative geometry, where the noncommutativity appears in a product of string fields. Later, four years ago, the Dirichlet branes (D-branes) have been recognized as solitonic objects in superstring theory [3]. Further it has been found that the low energy behavior of the D-branes are well described by supersymmetric Yang-Mills theory (SYM) [4]. In the situation of some D-branes coinciding, the space-time coordinates are promoted to matrices which appear as the fields in SYM. Then the size of the matrices corresponds to the number of the D-branes, so noncommutativity of the matrices is related to the noncommutative nature of space-time. On the other hand, it is known that when considering open strings in the presence of a constant background of the second-rank antisymmetric tensor field $B_{i j}$, the end points of the open strings become noncommutative [5, 6, 7]. It means a noncommutativity of the world volume coordinates of the D-branes, which occurs even for a single D-brane differently from the situation mentioned above. Although these two types of noncommutative natures have distinct origins, as was explicitly pointed out in refs. 8, 9], they are related to each other in the interesting way: "Infinitely many coincident $\mathrm{D} p$-branes can be described by a single $\mathrm{D}(p+2)$-brane under a constant $B$-field background."

Recently, Seiberg and Witten [10] discussed that the low energy behavior of the D-branes in the constant $B$-field background can be described either by commutative or noncommutative Yang-Mills theories?], which corresponds to the difference of the regularization scheme the Pauli-Villars regularization or the point-splitting regularization - adopted in the world sheet approach to open string theory. Also they argued the relation between the gauge field in the commutative theory and that in the noncommutative theory, and obtained its explicit form in some simple cases

In this paper we consider Witten's open string field theory in a constant background of the $B$-field. Then we should have a new noncommutativity originating form the $B$-field background in addition to the noncommutativity which already has appeared in the product of the string fields. We consider the open bosonic string field theory instead of supersymmetric one. Because the noncommutative structure mentioned above appears independently of supersymmetry, we do not consider the fermions for the sake of simplicity. We explicitly construct this open string theory by extending the operator formulation, which has been given by Gross and Jevicki [14, 15, 16] in the background of the flat space-time, to the case of the presence of the constant $B$-field background. As a result we obtain a product between the string fields added to the ordinary product by the Moyal product acting only on the zeromodes of the open strings, which reproduces the result of the correlation functions among

\footnotetext{
${ }^{2}$ The terminology "commutative Yang-Mills theory" or "noncommutative Yang-Mills theory" means that the geometry of the space-time where the Yang-Mills theory is defined on is commutative or noncommutative respectively.

${ }^{3}$ For related works, see refs. [11, 12, 13].
} 
vertex operators in the world sheet formulation [10]. It is noted that the part of the product concerning the nonzero-modes is not affected by the presence of the $B$-field background. The string field theory with the modified product gives noncommutative Yang-Mills theory in the low energy limit. In the string field theory perspective, there should exist a certain (hopefully simpler than the Yang-Mills case) transformation connecting the string field in the string field theory we have constructed and that in a string field theory leading to commutative Yang-Mills theory. Here we find that the Moyal type noncommutative structure can be absorbed by a unitary rotation of the string field. As a result of the rotation, the string field theory is transformed to a string field theory with the ordinary noncommutative product where the BRST charge in the kinetic term is unaffected. This fact suggests that the universality of the interaction vertices in various string field theories, which has been discussed in refs. [17, 18, 19, holds also between open string field theories with different boundary conditions caused by turning on the $B$-field. The string field theory obtained by the transformation can be considered to give a microscopic origin of the commutative Yang-Mills theory.

This paper is organized as follows. In section 2, we briefly review Witten's bosonic open string field theory and its explicit construction based on the operator formulation elaborated by Gross and Jevicki in the case of the Neumann boundary condition. We explain open strings in the presence of a constant $B$-field background and obtain the mode-expanded form of the open string in section 3. As a result, we see that in this situation the end points of the open string become noncommutative. It has already been discussed by many authors [5, 6, 0, 10]. But in order to make this paper more self-contained and to prepare for construction of Witten's string field theory in the constant $B$-field background, we give a somewhat detailed argument. In section 4 , based on the results in the previous sections, Witten's open string field theory under the constant $B$-field background is constructed explicitly by making use of the operator formulation. Here we obtain the structure of the Moyal product in addition to the ordinary noncommutativity which has already existed in the background of no $B$ field. In section 5, we find a certain unitary transformation of the string field which removes the Moyal type noncommutativity from the string field theory constructed in the previous section. This transformation can be essentially regarded as a microscopic analog of the map between the gauge fields in commutative and noncommutative Yang-Mills theories discussed in ref. [10]. In section 6, we also discuss about the background independence between open string field theories with different boundary conditions caused by the presence of the constant $B$-field. Until now we have not put the Chan-Paton factors to the end points of open strings, which corresponds to the case of the $U(1)$ gauge group in the Yang-Mills theories. However, in string field theory, it is quite a straightforward generalization to introduce the Chan-Paton factors as is pointed out in refs. [20, 21], so we can immediately have the results also in the case corresponding to the nonabelian gauge group. It forms a contrast to the situation in Yang-Mills theory, where in general it is difficult to obtain the concrete form of the map connecting the gauge fields of the commutative and noncommutative Yang-Mills theories except some simple casesf. Finally, section 7 is devoted to summary and discussion. In

\footnotetext{
${ }^{4}$ For example, the case of the $U(1)$ gauge fields giving constant field strengths.
} 
appendix A we present the result of the explicit forms of the overlap vertices discussed in section 2 .

\section{Brief Review of Witten's Open String Field Theory}

In this section, we review some basic properties of Witten's bosonic open string field theory [1] and its explicit construction based on a Fock space representation of string field functional and $\delta$-function overlap vertices [14, 15, 22].

\subsection{Witten's Open String Field Theory}

Witten introduced a beautiful formulation of open string field theory in terms of a noncommutative extension of differential geometry, where string fields, the BRST operator $Q$ and the integration over the string configurations $\int$ in string field theory are analogs of differetial forms, the exterior derivative $d$ and the integration over the manifold $\int_{M}$ in the differential geometry, respectively. The ghost number assigned to the string field corresponds to the degree of the differential form. Also the (noncommutative) product between the string fields $*$ is interpreted as an analog of the wedge product $\wedge$ between the differential forms.

The axioms obeyed by the system of $\int, *$ and $Q$ are

$$
\begin{aligned}
& \int Q A=0, \\
& Q(A * B)=(Q A) * B+(-1)^{n_{A}} A *(Q B), \\
& (A * B) * C=A *(B * C), \\
& \int A * B=(-1)^{n_{A} n_{B}} \int B * A,
\end{aligned}
$$

where $A, B$ and $C$ are arbitrary string fields, whose ghost number is half-integer valued: The ghost number of $A$ is defined by the integer $n_{A}$ as $n_{A}+\frac{1}{2}$.

Then Witten discussed the following string field theory action

$$
S=\frac{1}{G_{s}} \int\left(\frac{1}{2} \psi * Q \psi+\frac{1}{3} \psi * \psi * \psi\right)
$$

where $G_{s}$ is the open string coupling constant and $\psi$ is the string field with the ghost number $-\frac{1}{2}$. The action is invariant under the gauge transformation

$$
\delta \psi=Q \Lambda+\psi * \Lambda-\Lambda * \psi,
$$

with the gauge parameter $\Lambda$ of the ghost number $-\frac{3}{2}$.

\subsection{Operator Formulation of String Field Theory}

The objects defined above can be explicitly constructed by using the operator formulation, where the string field is represented as a Fock space, and the integration $\int$ as an inner 
product on the Fock space. It was considered by Gross and Jevicki [14, 15] in the case of the Neumann boundary condition. We will heavily use the notation of the papers [14, 15]. In the operator formulation, the action (2.2) is described as

$$
S=\frac{1}{G_{s}}\left(\frac{1}{2}{ }_{12}\left\langle V_{2}|| \psi\right\rangle_{1} Q|\psi\rangle_{2}+\frac{1}{3}{ }_{123}\left\langle V_{3}|| \psi\right\rangle_{1}|\psi\rangle_{2}|\psi\rangle_{3}\right)
$$

where the structure of the product $*$ in the kinetic and potential terms is encoded to that of the overlap vertices $\left\langle V_{2}\right|$ and $\left\langle V_{3}\right|$ respectively?.

As a preparation for giving the explicit form of the overlaps, let us consider open strings in 26-dimensional space-time with the constant metric $G_{i j}$ in the Neumann boundary condition. The world sheet action is given by

$$
S_{W S}=\frac{1}{4 \pi \alpha^{\prime}} \int d \tau \int_{0}^{\pi} d \sigma G_{i j}\left(\partial_{\tau} X^{i} \partial_{\tau} X^{j}-\partial_{\sigma} X^{i} \partial_{\sigma} X^{j}\right)+S_{g h},
$$

where $S_{g h}$ is the action of the $b c$-ghosts:

$$
S_{g h}=\frac{i}{2 \pi} \int d \tau \int_{0}^{\pi} d \sigma\left[c_{+}\left(\partial_{\tau}-\partial_{\sigma}\right) b_{+}+c_{-}\left(\partial_{\tau}+\partial_{\sigma}\right) b_{-}\right] .
$$

Under the Neumann boundary condition, the string coordinates have the standard mode expansions:

$$
X^{j}(\tau, \sigma)=x^{j}+2 \alpha^{\prime} \tau p^{j}+i \sqrt{2 \alpha^{\prime}} \sum_{n \neq 0} \frac{1}{n} \alpha_{n}^{j} e^{-i n \tau} \cos (n \sigma),
$$

also the mode expansions of the ghosts are given by

$$
\begin{aligned}
& c_{ \pm}(\tau, \sigma)=\sum_{n \in \mathbf{Z}} c_{n} e^{-i n(\tau \pm \sigma)} \equiv c(\tau, \sigma) \pm i \pi_{b}(\tau, \sigma), \\
& b_{ \pm}(\tau, \sigma)=\sum_{n \in \mathbf{Z}} b_{n} e^{-i n(\tau \pm \sigma)} \equiv \pi_{c}(\tau, \sigma) \mp i b(\tau, \sigma) .
\end{aligned}
$$

As a result of the quantization, the modes obey the commutation relatons:

$$
\begin{aligned}
& {\left[x^{i}, p^{j}\right]=i G^{i j}, \quad\left[\alpha_{n}^{i}, \alpha_{m}^{j}\right]=n G^{i j} \delta_{n+m, 0},} \\
& \left\{b_{n}, c_{m}\right\}=\delta_{n+m, 0},
\end{aligned}
$$

the otherwises vanish.

The overlap

$$
\left|V_{N}\right\rangle=\left|V_{N}\right\rangle^{X}\left|V_{N}\right\rangle^{g h} \quad(N=1,2, \cdots)
$$

is the state satisfying the continuity conditions for the string coordinates and the ghosts at the $N$-string vertex of the string field theory. The superscripts $X$ and $g h$ show the

\footnotetext{
${ }^{5}$ Subscripts put to vectors in the Fock space label the strings concerning the vertices.
} 
contribution of the sectors of the coordinates and the ghosts respectively. The continuity conditions for the coordinates are

$$
\begin{aligned}
& \left(X^{(r) j}(\sigma)-X^{(r-1) j}(\pi-\sigma)\right)\left|V_{N}\right\rangle^{X}=0 \\
& \left(P_{i}^{(r)}(\sigma)+P_{i}^{(r-1)}(\pi-\sigma)\right)\left|V_{N}\right\rangle^{X}=0
\end{aligned}
$$

for $0 \leq \sigma \leq \frac{\pi}{2}$ and $r=1, \cdots, N$. Here $P_{i}(\sigma)$ is the momentum conjugate to the coordinate $X^{j}(\sigma)$ at $\tau=0$, and the superscript $(r)$ labels the string $(r)$ meeting at the vertex $\sigma$. In the above formulas, we regard $r=0$ as $r=N$ because of the cyclic property of the vertex. For the ghost sector, we impose the following conditions on the variables $c(\sigma), b(\sigma)$ and their conjugate momenta $\pi_{c}(\sigma), \pi_{b}(\sigma)$ :

$$
\begin{aligned}
& \left(\pi_{c}^{(r)}(\sigma)-\pi_{c}^{(r-1)}(\pi-\sigma)\right)\left|V_{N}\right\rangle^{g h}=0, \\
& \left(b^{(r)}(\sigma)-b^{(r-1)}(\pi-\sigma)\right)\left|V_{N}\right\rangle^{g h}=0, \\
& \left(c^{(r)}(\sigma)+c^{(r-1)}(\pi-\sigma)\right)\left|V_{N}\right\rangle^{g h}=0, \\
& \left(\pi_{b}^{(r)}(\sigma)+\pi_{b}^{(r-1)}(\pi-\sigma)\right)\left|V_{N}\right\rangle^{g h}=0,
\end{aligned}
$$

for $0 \leq \sigma \leq \frac{\pi}{2}$ and $r=1, \cdots, N$.

We present the explicit form of $\left|V_{N}\right\rangle$ for $N=1,2,3,4$ in appendix A.

\section{Open Strings in Constant B-Field Background}

We consider a constant background of the second-rank antisymmetric tensor field $B_{i j}$ in addition to the constant metric $g_{i j}$ where open strings propagate. Then the boundary conditon at the end points of the open strings changes from the Neumann type, and thus the open string has a different mode expansion from the Neumann case (2.7). As a result, the end point is to be noncommutative, in the picture of the D-branes which implies noncommutativity of the world volume coordinates on the D-branes. Here we derive the mode-expanded form of the open string coordinates as a preparation for a calculation of the overlap vertices in the next section.

We start with the world sheet action

$$
\begin{aligned}
S_{W S}^{B}= & \frac{1}{4 \pi \alpha^{\prime}} \int d \tau \int_{0}^{\pi} d \sigma\left[g_{i j}\left(\partial_{\tau} X^{i} \partial_{\tau} X^{j}-\partial_{\sigma} X^{i} \partial_{\sigma} X^{j}\right)-2 \pi \alpha^{\prime} B_{i j}\left(\partial_{\tau} X^{i} \partial_{\sigma} X^{j}-\partial_{\sigma} X^{i} \partial_{\tau} X^{j}\right)\right] \\
& +S_{g h} .
\end{aligned}
$$

Because the term proportional to $B_{i j}$ can be written as a total derivative term, it does not affect the equation of motion but does the boundary condition, which requires

$$
g_{i j} \partial_{\sigma} X^{j}-2 \pi \alpha^{\prime} B_{i j} \partial_{\tau} X^{j}=0
$$

\footnotetext{
${ }^{6}$ We often use the expression $\left|V_{N}\right\rangle_{12 \cdots N}$ for the overlap when specifying the strings concerning the vertex.
} 
on $\sigma=0, \pi$. This can be rewritten to the convenient form

$$
E_{i j} \partial_{-} X^{j}=\left(E^{T}\right)_{i j} \partial_{+} X^{j}
$$

where $E_{i j} \equiv g_{i j}+2 \pi \alpha^{\prime} B_{i j}$, and $\partial_{ \pm}$are derivatives with respect to the light cone variables $\sigma^{ \pm}=\tau \pm \sigma$. We can easily see that $X^{j}(\tau, \sigma)$ satisfying the boundary condition (3.3) has the following mode expansion:

$$
\begin{aligned}
X^{j}(\tau, \sigma)= & \tilde{x}^{j}+\alpha^{\prime}\left[\left(E^{-1}\right)^{j k} g_{k l} p^{l} \sigma^{-}+\left(E^{-1 T}\right)^{j k} g_{k l} p^{l} \sigma^{+}\right] \\
& +i \sqrt{\frac{\alpha^{\prime}}{2}} \sum_{n \neq 0} \frac{1}{n}\left[\left(E^{-1}\right)^{j k} g_{k l} \alpha_{n}^{l} e^{-i n \sigma^{-}}+\left(E^{-1 T}\right)^{j k} g_{k l} \alpha_{n}^{l} e^{-i n \sigma^{+}}\right] .
\end{aligned}
$$

We will obtain the commutators between the modes from the propagator of the open strings, which gives another derivation different from the method by $\mathrm{Chu}$ and Ho [6] based on the quantization via the Dirac bracket. When performing the Wick rotation $\tau \rightarrow-i \tau$ and mapping the world sheet to the upper half plane $z=e^{\tau+i \sigma}, \bar{z}=e^{\tau-i \sigma}(0 \leq \sigma \leq \pi)$, the boundary condition (3.3) becomes

$$
E_{i j} \partial_{\bar{z}} X^{j}=\left(E^{T}\right)_{i j} \partial_{z} X^{j}
$$

which is imposed on the real axis $z=\bar{z}$. The propagator $\left\langle X^{i}(z, \bar{z}) X^{j}\left(z^{\prime}, \overline{z^{\prime}}\right)\right\rangle$ satisfying the boundary condition (3.5) is determined as

$$
\begin{aligned}
\left\langle X^{i}(z, \bar{z}) X^{j}\left(z^{\prime}, \overline{z^{\prime}}\right)\right\rangle= & -\alpha^{\prime}\left[g^{i j} \ln \left|z-z^{\prime}\right|-g^{i j} \ln \left|z-\overline{z^{\prime}}\right|\right. \\
& \left.+G^{i j} \ln \left|z-\bar{z}^{\prime}\right|^{2}+\frac{1}{2 \pi \alpha^{\prime}} \theta^{i j} \ln \frac{z-\bar{z}^{\prime}}{\bar{z}-z^{\prime}}+D^{i j}\right],
\end{aligned}
$$

where $G^{i j}$ and $\theta^{i j}$ are given by

$$
\begin{aligned}
G^{i j} & =\frac{1}{2}\left(E^{T-1}+E^{-1}\right)^{i j}=\left(E^{T-1} g E^{-1}\right)^{i j}=\left(E^{-1} g E^{T-1}\right)^{i j} \\
\theta^{i j} & =2 \pi \alpha^{\prime} \cdot \frac{1}{2}\left(E^{T-1}-E^{-1}\right)^{i j} \\
& =\left(2 \pi \alpha^{\prime}\right)^{2}\left(E^{T-1} B E^{-1}\right)^{i j}=-\left(2 \pi \alpha^{\prime}\right)^{2}\left(E^{-1} B E^{T-1}\right)^{i j}
\end{aligned}
$$

Also the constant $D^{i j}$ remains unknown from the boundary condition alone. However it is an irrelevant parameter, so we can fix an appropriate value. The mode-expanded form (3.4) is mapped to

$$
\begin{aligned}
X^{j}(z, \bar{z})= & \tilde{x}^{j}-i \alpha^{\prime}\left[\left(E^{-1}\right)^{j k} p_{k} \ln \bar{z}+\left(E^{-1 T}\right)^{j k} p_{k} \ln z\right] \\
& +i \sqrt{\frac{\alpha^{\prime}}{2}} \sum_{n \neq 0} \frac{1}{n}\left[\left(E^{-1}\right)^{j k} \alpha_{n, k} \bar{z}^{-n}+\left(E^{-1 T}\right)^{j k} \alpha_{n, k} z^{-n}\right] .
\end{aligned}
$$


Note that the indices of $p^{l}$ and $\alpha_{n}^{l}$ were lowered by the metric $g_{i j}$ not $G_{i j}$. Recall the definition of the propagator

$$
\left\langle X^{i}(z, \bar{z}) X^{j}\left(z^{\prime}, \overline{z^{\prime}}\right)\right\rangle \equiv R\left(X^{i}(z, \bar{z}) X^{j}\left(z^{\prime}, \overline{z^{\prime}}\right)\right)-N\left(X^{i}(z, \bar{z}) X^{j}\left(z^{\prime}, \overline{z^{\prime}}\right)\right)
$$

where $R$ and $N$ stand for the radial ordering and the normal ordering respectively. We take a prescription for the normal ordering which pushes $p_{i}$ to the right and $\tilde{x}_{j}$ to the left with respect to the zero-modes $p_{i}$ and $\tilde{x}_{j}$. It corresponds to considering the vacuum satisfying

$$
p_{j}|0\rangle=\alpha_{n, j}|0\rangle=0 \quad(n>0), \quad\langle 0| \alpha_{n, j}=0 \quad(n<0),
$$

which is the standard prescription for calculating the propagator of the massless scalar field in two-dimensional conformal field theory from the operator formalism. Making use of eqs. (3.10), (3.11) and techniques of the contour integration, it is easy to obtain the commutators

$$
\left[\alpha_{n, i}, \alpha_{m, j}\right]=n \delta_{n+m, 0} G_{i j}, \quad\left[\tilde{x}^{i}, p_{j}\right]=i \delta_{j}^{i},
$$

where the first equation holds for all integers with $\alpha_{0, i} \equiv \sqrt{2 \alpha^{\prime}} p_{i}$. The constant $D^{i j}$ is written as $\alpha^{\prime} D^{i j}=-\left\langle 0\left|\tilde{x}^{i} \tilde{x}^{j}\right| 0\right\rangle$, which corresponds to define the normal ordering with respect to $\tilde{x}^{i}$ s as

$$
: \tilde{x}^{i} \tilde{x}^{j}:=\tilde{x}^{i} \tilde{x}^{j}+\alpha^{\prime} D^{i j} .
$$

Let us fix $D^{i j}$ as $\alpha^{\prime} D^{i j}=-\frac{i}{2} \theta^{i j}$, which is the convention taken in the paper [10]. Then the coordinates $\tilde{x}^{i}$ become noncommutative:

$$
\left[\tilde{x}^{i}, \tilde{x}^{j}\right]=i \theta^{i j}
$$

but the center of mass coordinates $x^{i} \equiv \tilde{x}^{i}+\frac{1}{2} \theta^{i j} p_{j}$ can be seen to commute each other.

Now we have the mode-expanded form of the string coordinates and the commutation relations between the modes, which are

$$
\begin{aligned}
X^{j}(\tau, \sigma)= & x^{j}+2 \alpha^{\prime}\left(G^{j k} \tau+\frac{1}{2 \pi \alpha^{\prime}} \theta^{j k}\left(\sigma-\frac{\pi}{2}\right)\right) p_{k} \\
& +i \sqrt{2 \alpha^{\prime}} \sum_{n \neq 0} \frac{1}{n} e^{-i n \tau}\left[G^{j k} \cos (n \sigma)-i \frac{1}{2 \pi \alpha^{\prime}} \theta^{j k} \sin (n \sigma)\right] \alpha_{n, k}, \\
& {\left[\alpha_{n, i}, \alpha_{m, j}\right]=n \delta_{n+m, 0} G_{i j}, \quad\left[x^{i}, p_{j}\right]=i \delta_{j}^{i}, }
\end{aligned}
$$

with all the other commutators vanishing.

Also, due to the formula

$$
\sum_{n=1}^{\infty} \frac{2}{n} \sin \left(n\left(\sigma+\sigma^{\prime}\right)\right)= \begin{cases}\pi-\sigma-\sigma^{\prime} & \left(\sigma+\sigma^{\prime} \neq 0,2 \pi\right) \\ 0 & \left(\sigma+\sigma^{\prime}=0,2 \pi\right),\end{cases}
$$

we can see by a direct calculation that the end points of the string become noncommutative

$$
\left[X^{i}(\tau, \sigma), X^{j}\left(\tau, \sigma^{\prime}\right)\right]= \begin{cases}i \theta^{i j} & \left(\sigma=\sigma^{\prime}=0\right) \\ -i \theta^{i j} & \left(\sigma=\sigma^{\prime}=\pi\right) \\ 0 & \text { (otherwises) }\end{cases}
$$


On the other hand, it is noted that the conjugate momenta have the mode expansion identical with that in the Neumann case:

$$
\begin{aligned}
P_{i}(\tau, \sigma) & =\frac{1}{2 \pi \alpha^{\prime}}\left(g_{i j} \partial_{\tau}-2 \pi \alpha^{\prime} B_{i j} \partial_{\sigma}\right) X^{j}(\tau, \sigma) \\
& =\frac{1}{\pi} p_{i}+\frac{1}{\pi \sqrt{2 \alpha^{\prime}}} \sum_{n \neq 0} e^{-i n \tau} \cos (n \sigma) \alpha_{n, i} .
\end{aligned}
$$

At the end of this section, we remark that the relations (3.7) and (3.8) are in the same form as a T-duality transformation, although the correspondence is a formal sense, because we are not considering any compactification of space-time. The generalized T-duality transformation, namely $O(D, D)$-transformation, is defined by

$$
E^{\prime}=(a E+b)(c E+d)^{-1}
$$

with $a, b, c$ and $d$ being $D \times D$ real matrices. ( $D$ is the dimension of space-time.) The matrix

$$
h=\left(\begin{array}{ll}
a & b \\
c & d
\end{array}\right)
$$

is $O(D, D)$ matrix, which satisfies

$$
h^{T} J h=J
$$

where

$$
J=\left(\begin{array}{cc}
0 & \mathbf{1}_{D} \\
\mathbf{1}_{D} & 0
\end{array}\right)
$$

The relations (3.7) and (3.8) correspond to the case of the inversion $a=d=0, b=c=\mathbf{1}_{D}$.

\section{Construction of Overlap Vertices}

Here we construct Witten's open string theory in the constant $B$-field background by obtaining the explicit formulas of the overlap vertices. As is understood from the fact that the action of the ghosts (2.6) contains no background fields, the ghost sector is not affected by turning on the $B$-field background. Thus we may consider the coordinate sector only. First, let us see the mode-expanded forms of the coordinates and the momenta at $\tau=0$

$$
\begin{aligned}
X^{j}(\sigma)= & G^{j k} y_{k}+\frac{1}{\pi} \theta^{j k}\left(\sigma-\frac{\pi}{2}\right) p_{k} \\
& +2 \sqrt{\alpha^{\prime}} \sum_{n=1}^{\infty}\left[G^{j k} \cos (n \sigma) x_{n, k}+\frac{1}{2 \pi \alpha^{\prime}} \theta^{j k} \sin (n \sigma) \frac{1}{n} p_{n, k}\right] \\
P_{i}(\sigma)= & \frac{1}{\pi} p_{i}+\frac{1}{\pi \sqrt{\alpha^{\prime}}} \sum_{n=1}^{\infty} \cos (n \sigma) p_{n, i},
\end{aligned}
$$


where $x^{j}=G^{j k} y_{k}$, the coordinates and the momenta for the oscillator modes are

$$
\begin{aligned}
& x_{n, k}=\frac{i}{2} \sqrt{\frac{2}{n}}\left(a_{n, k}-a_{n, k}^{\dagger}\right)=\frac{i}{\sqrt{2} n}\left(\alpha_{n, k}-\alpha_{-n, k}\right), \\
& p_{n, k}=\sqrt{\frac{n}{2}}\left(a_{n, k}+a_{n, k}^{\dagger}\right)=\frac{1}{\sqrt{2}}\left(\alpha_{n, k}+\alpha_{-n, k}\right) .
\end{aligned}
$$

The nonvanishing commutators are given by

$$
\left[x_{n, k}, p_{m, l}\right]=i G_{k l} \delta_{n, m}, \quad\left[y_{k}, p_{l}\right]=i G_{k l} .
$$

We should note that the metric appearing in eqs. (4.4) is $G_{i j}$, instead of $g_{i j}$. So it can be seen that if we employ the variables with the lowered space-time indices $y_{k}, p_{k}, x_{n, k}$ and $p_{n, k}$, the metric used in the expression of the overlaps is $G^{i j}$ not $g^{i j}$.

The continuity condition (2.11) is universal for any background, and the mode expansion of the momenta $P_{i}(\sigma)$ 's is of the same form as in the Neumann case, thus the continuity conditions for the momenta in terms of the modes $p_{n, i}$ are identical with those in the Neumann case. Also, since $p_{n, i}$ 's mutually commute, it is natural to find a solution of the continuity condition, assuming the following form for the overlap vertices:

$$
\left|\hat{V}_{N}\right\rangle_{1 \cdots N}^{X}=\exp \left[\frac{i}{4 \pi \alpha} \theta^{i j} \sum_{r, s=1}^{N} p_{n, i}^{(r)} Z_{n m}^{r s} p_{m, j}^{(s)}\right]\left|V_{N}\right\rangle_{1 \cdots N}^{X}
$$

where $\left|\hat{V}_{N}\right\rangle_{1 \cdots N}^{X}$ and $\left|V_{N}\right\rangle_{1 \cdots N}^{X}$ are the overlaps in the background corresponding to the world sheet actions (3.1) and (2.5) respectively, the explicit form of the latter is given in appendix A. Clearly the expression (4.5) satisfies the continuity conditions for the modes of the momenta, and the coefficients $Z_{n m}^{r s}$ 's are determined so that the continuity conditions for the coordinates are satisfied.

\section{1 $|\hat{I}\rangle^{X} \equiv\left|\hat{V}_{1}\right\rangle^{X}$}

For the $N=1$ case, we consider the identity overlap $|\hat{I}\rangle^{X} \equiv\left|\hat{V}_{1}\right\rangle^{X}$. The continuity conditions for the momenta require that

$$
P_{i}(\sigma)+P_{i}(\pi-\sigma)=\frac{2}{\pi} p_{i}+\frac{2}{\pi \sqrt{\alpha^{\prime}}} \sum_{n=2,4,6, \cdots} \cos (n \sigma) p_{n, i}
$$

should vanish for $0 \leq \sigma \leq \frac{\pi}{2}$, namely,

$$
p_{i}=0, \quad p_{n, i}=0 \quad(n=2,4,6, \cdots),
$$

which is satisfied by the overlap in the Neumann case $|I\rangle$. In addition, the conditions for the coordinates are that

$$
X^{j}(\sigma)-X^{j}(\pi-\sigma)=\frac{2}{\pi} \theta^{j k}\left(\sigma-\frac{\pi}{2}\right) p_{k}
$$




$$
\begin{aligned}
& +4 \sqrt{\alpha^{\prime}} \sum_{n=1,3,5, \cdots} G^{j k} \cos (n \sigma) x_{n, k} \\
& +4 \sqrt{\alpha^{\prime}} \sum_{n=2,4,6, \cdots} \frac{1}{2 \pi \alpha^{\prime}} \theta^{j k} \sin (n \sigma) \frac{1}{n} p_{n, k}
\end{aligned}
$$

should vanish for $0 \leq \sigma \leq \frac{\pi}{2}$. The first and third lines in the r. h. s. can be put to zero by using eq. (4.7). So what we have to consider is the remaining condition $x_{n, k}=0$ for $n=1,3,5, \cdots$, which however is nothing but the continuity condition for the coordinates in the Neumann case. It can be understood from the point that the second line in eq. (4.8) does not depend on $\theta^{i j}$. Thus it turns out that the continuity conditions in the case of the $B$-field turned on are satisfied by the identity overlap made in the Neumann case (A.1). The solution is

$$
|\hat{I}\rangle^{X}=|I\rangle^{X}=\exp \left[-\frac{1}{2} G^{i j} \sum_{n=0}^{\infty}(-1)^{n} a_{n, i}^{\dagger} a_{n, j}^{\dagger}\right]|0\rangle,
$$

where also the zero modes $y_{i}$ and $p_{i}$ are written by using the creation and annihilation operators $a_{0, i}^{\dagger}$ and $a_{0, i}$ as

$$
y_{i}=\frac{i}{2} \sqrt{2 \alpha^{\prime}}\left(a_{0, i}-a_{0, i}^{\dagger}\right), \quad p_{i}=\frac{1}{\sqrt{2 \alpha^{\prime}}}\left(a_{0, i}+a_{0, i}^{\dagger}\right) .
$$

\section{$4.2 \quad\left|\hat{V}_{2}\right\rangle_{12}^{X}$}

For the $N=2$ case, we are to do the same argument as in the $N=1$ case. The continuity conditions mean that

$$
\begin{aligned}
P_{i}^{(1)}(\sigma)+P_{i}^{(2)}(\pi-\sigma)= & \frac{1}{\pi}\left(p_{i}^{(1)}+p_{i}^{(2)}\right)+\frac{1}{\pi \sqrt{\alpha^{\prime}}} \sum_{n=1}^{\infty} \cos (n \sigma)\left(p_{n, i}^{(1)}+(-1)^{n} p_{n, i}^{(2)}\right) \\
X^{(1) j}(\sigma)-X^{(2) j}(\pi-\sigma)= & G^{j k}\left(y_{k}^{(1)}-y_{k}^{(2)}\right)+\frac{1}{\pi} \theta^{j k}\left(\sigma-\frac{\pi}{2}\right)\left(p_{k}^{(1)}+p_{k}^{(2)}\right) \\
+2 \sqrt{\alpha^{\prime}} \sum_{n=1}^{\infty}[ & G^{j k} \cos (n \sigma)\left(x_{n, k}^{(1)}-(-1)^{n} x_{n, k}^{(2)}\right) \\
& \left.+\frac{1}{2 \pi \alpha^{\prime}} \theta^{j k} \sin (n \sigma) \frac{1}{n}\left(p_{n, k}^{(1)}+(-1)^{n} p_{n, k}^{(2)}\right)\right]
\end{aligned}
$$

should be zero for $0 \leq \sigma \leq \pi$. It turns out again that the conditions for the modes are identical with those in the Neumann case:

$$
\begin{array}{ll}
p_{i}^{(1)}+p_{i}^{(2)}=0, & p_{n, i}^{(1)}+(-1)^{n} p_{n, i}^{(2)}=0, \\
y_{i}^{(1)}-y_{i}^{(2)}=0, & x_{n, i}^{(1)}-(-1)^{n} x_{n, i}^{(2)}=0
\end{array}
$$


for $n \geq 1$. Thus we have the solution ${ }^{7}$

$$
\left|\hat{V}_{2}\right\rangle_{12}^{X}=\left|V_{2}\right\rangle_{12}^{X}=\exp \left[-G^{i j} \sum_{n=0}^{\infty}(-1)^{n} a_{n, i}^{(1) \dagger} a_{n, j}^{(2) \dagger}\right]|0\rangle_{12} .
$$

\section{$4.3 \quad\left|\hat{V}_{4}\right\rangle_{1234}^{X}$}

We find a solution of the continuity conditions (2.11) in the $N=4$ case assuming the form

$$
\left|\hat{V}_{4}\right\rangle_{1234}^{X}=\exp \left[\frac{i}{4 \pi \alpha} \theta^{i j} \sum_{r, s=1}^{4} p_{n, i}^{(r)} Z_{n m}^{r s} p_{m, j}^{(s)}\right]\left|V_{4}\right\rangle_{1234}^{X} .
$$

When considering the continuity conditions, it is convenient to employ the $Z_{4}$-Fourier transformed variables:

$$
\begin{aligned}
Q_{1}^{j}(\sigma) & =\frac{1}{2}\left[i X^{(1) j}(\sigma)-X^{(2) j}(\sigma)-i X^{(3) j}(\sigma)+X^{(4) j}(\sigma)\right] \equiv Q^{j}(\sigma), \\
Q_{2}^{j}(\sigma) & =\frac{1}{2}\left[-X^{(1) j}(\sigma)+X^{(2) j}(\sigma)-X^{(3) j}(\sigma)+X^{(4) j}(\sigma)\right], \\
Q_{3}^{j}(\sigma) & =\frac{1}{2}\left[-i X^{(1) j}(\sigma)-X^{(2) j}(\sigma)+i X^{(3) j}(\sigma)+X^{(4) j}(\sigma)\right] \equiv \bar{Q}^{j}(\sigma), \\
Q_{4}^{j}(\sigma) & =\frac{1}{2}\left[X^{(1) j}(\sigma)+X^{(2) j}(\sigma)+X^{(3) j}(\sigma)+X^{(4) j}(\sigma)\right] .
\end{aligned}
$$

For the momentum variables we also define the $Z_{4}$-Fourier transformed variables $P_{1, i}(\sigma)(\equiv$ $\left.P_{i}(\sigma)\right), P_{2, i}(\sigma), P_{3, i}(\sigma)\left(\equiv \bar{P}_{i}(\sigma)\right)$ and $P_{4, i}(\sigma)$ by the same combinations of $P_{i}^{(r)}(\sigma)$ 's as the above. These variables have the following mode expansions

$$
\begin{aligned}
P_{t, i}(\sigma)= & \frac{1}{\pi \sqrt{2 \alpha^{\prime}}} P_{t, 0, i}+\frac{1}{\pi \sqrt{\alpha^{\prime}}} \sum_{n=1}^{\infty} \cos (n \sigma) P_{t, n, i} \\
Q_{t}^{j}(\sigma)= & G^{j k \sqrt{2 \alpha^{\prime}}} Q_{t, 0, k}+\frac{1}{\pi} \theta^{j k}\left(\sigma-\frac{\pi}{2}\right) \frac{1}{\sqrt{2 \alpha^{\prime}}} P_{t, 0, k} \\
& +\sqrt{2 \alpha^{\prime}} \sum_{n=1}^{\infty}\left[G^{j k} \cos (n \sigma) Q_{t, n, k}+\frac{1}{2 \pi \alpha^{\prime}} \theta^{j k} \sin (n \sigma) \frac{1}{n} P_{t, n, k}\right],
\end{aligned}
$$

where $t=1,2,3,4$. From now on, we frequently omit the subscript $t$ for the $t=1$ case, and at the same time we employ the expression with a bar instead of putting the subscript $t$ for the $t=3$ case.

Using those variables, the continuity conditions are written as

$$
Q_{4}^{j}(\sigma)-Q_{4}^{j}(\pi-\sigma)=0, \quad P_{4, i}(\sigma)+P_{4, i}(\pi-\sigma)=0,
$$

\footnotetext{
${ }^{7}$ For the $N=1$ and 2 cases, it turns out that the phase factor in $(4.5)$ becomes trivial, by using the continuity conditions for the momenta and the antisymmetric property of $\theta^{i j}$. We can understand the results (4.9) and (4.14) also from this point.
} 


$$
\begin{aligned}
Q_{2}^{j}(\sigma)+Q_{2}^{j}(\pi-\sigma) & =0, & & P_{2, i}(\sigma)-P_{2, i}(\pi-\sigma)=0, \\
Q^{j}(\sigma)-i Q^{j}(\pi-\sigma) & =0, & & P_{i}(\sigma)+i P_{i}(\pi-\sigma)=0, \\
\bar{Q}^{j}(\sigma)+i \bar{Q}^{j}(\pi-\sigma) & =0, & & \bar{P}_{i}(\sigma)-i \bar{P}_{i}(\pi-\sigma)=0
\end{aligned}
$$

for $0 \leq \sigma \leq \frac{\pi}{2}$. In terms of the modes, the conditions for the sectors of $t=2$ and 4 are identical with the Neumann case

$$
\begin{aligned}
& \left.\left.(1-C) \mid Q_{4, k}\right)\left|\hat{V}_{4}\right\rangle^{X}=(1+C) \mid P_{4, k}\right)\left|\hat{V}_{4}\right\rangle^{X}=0, \\
& \left.\left.(1+C) \mid Q_{2, k}\right)\left|\hat{V}_{4}\right\rangle^{X}=(1-C) \mid P_{2, k}\right)\left|\hat{V}_{4}\right\rangle^{X}=0,
\end{aligned}
$$

which can be seen from the point that the conditions (4.18) for the sectors of $t=2$ and 4 lead the same relations between the modes as those without the terms containing $\theta^{j k}$. Here we adopted the vector notation for the modes

$$
\left.\left.\mid Q_{t, k}\right)=\left[\begin{array}{c}
Q_{t, 0, k} \\
Q_{t, 1, k} \\
\vdots
\end{array}\right], \quad \mid P_{t, k}\right)=\left[\begin{array}{c}
P_{t, 0, k} \\
P_{t, 1, k} \\
\vdots
\end{array}\right]
$$

and $C$ is a matrix such that $(C)_{n m}=(-1)^{n} \delta_{n m}(n, m \geq 0)$. Thus there is needed no correction containing $\theta^{i j}$ for the sectors of $t=2$ and 4 , so it is natural to assume the form of the phase factor in eq. (4.15) as

$$
\frac{1}{2} \theta^{i j} \sum_{r, s=1}^{4}\left(p_{i}^{(r)}\left|Z^{r s}\right| p_{j}^{(s)}\right)=\theta^{i j}\left(P_{i}|Z| \bar{P}_{j}\right)
$$

with $Z$ being anti-hermitian.

Next let us consider the conditions for the sectors of $t=1$ and 3 . We rewrite the mode expansions of $Q^{j}(\sigma)$ and $\bar{Q}^{j}(\sigma)$ as

$$
\begin{aligned}
Q^{j}(\sigma)= & G^{j k}\left(\sqrt{2 \alpha^{\prime}} Q_{0, k}+2 \sqrt{\alpha^{\prime}} \sum_{n=1}^{\infty} \cos (n \sigma) Q_{n, k}\right) \\
& +\theta^{j k}\left[\int_{\pi / 2}^{\sigma} d \sigma^{\prime} P_{i}\left(\sigma^{\prime}\right)+\frac{1}{\pi \sqrt{\alpha^{\prime}}} \sum_{n=1,3,5, \cdots} \frac{1}{n}(-1)^{(n-1) / 2} P_{n, k}\right] \\
\equiv & \theta^{j k} \int_{\pi / 2}^{\sigma} d \sigma^{\prime} P_{i}\left(\sigma^{\prime}\right)+\Delta Q^{j}(\sigma), \\
\bar{Q}^{j}(\sigma)= & G^{j k}\left(\sqrt{2 \alpha^{\prime}} \bar{Q}_{0, k}+2 \sqrt{\alpha^{\prime}} \sum_{n=1}^{\infty} \cos (n \sigma) \bar{Q}_{n, k}\right) \\
& +\theta^{j k}\left[\int_{\pi / 2}^{\sigma} d \sigma^{\prime} \bar{P}_{i}\left(\sigma^{\prime}\right)+\frac{1}{\pi \sqrt{\alpha^{\prime}}} \sum_{n=1,3,5, \cdots} \frac{1}{n}(-1)^{(n-1) / 2} \bar{P}_{n, k}\right] \\
\equiv & \theta^{j k} \int_{\pi / 2}^{\sigma} d \sigma^{\prime} \bar{P}_{i}\left(\sigma^{\prime}\right)+\Delta \bar{Q}^{j}(\sigma) .
\end{aligned}
$$


Using the conditions for $P_{i}(\sigma)$ and $\bar{P}_{i}(\sigma)$ in (4.18), we can reduce the conditions for $Q^{j}(\sigma)$ and $\bar{Q}^{j}(\sigma)$ to those for $\Delta Q^{j}(\sigma)$ and $\Delta \bar{Q}^{j}(\sigma)$ :

$$
\begin{aligned}
& \Delta Q^{j}(\sigma)= \begin{cases}i \Delta Q^{j}(\pi-\sigma) & \left(0 \leq \sigma \leq \frac{\pi}{2}\right) \\
-i \Delta Q^{j}(\pi-\sigma) & \left(\frac{\pi}{2} \leq \sigma \leq \pi\right),\end{cases} \\
& \Delta \bar{Q}^{j}(\sigma)= \begin{cases}-i \Delta \bar{Q}^{j}(\pi-\sigma) & \left(0 \leq \sigma \leq \frac{\pi}{2}\right) \\
i \Delta \bar{Q}^{j}(\pi-\sigma) & \left(\frac{\pi}{2} \leq \sigma \leq \pi\right) .\end{cases}
\end{aligned}
$$

These formulas are translated to the relations between the modes via the Fourier transformation. The result is expressed in the vector notation as

$$
\left.\left.(1-X) \mid \mathcal{Q}_{i}\right)\left|\hat{V}_{4}\right\rangle^{X}=(1+X) \mid \overline{\mathcal{Q}}_{i}\right)\left|\hat{V}_{4}\right\rangle^{X}=0,
$$

where the matrix $X$ is defined by eqs. (A.16) and (A.17), also the vectors $\mid \mathcal{Q}_{i}$ ) and $\mid \overline{\mathcal{Q}}_{i}$ ) stand for

$$
\begin{aligned}
\left.\mid \mathcal{Q}_{i}\right)= & {\left[\begin{array}{c}
Q_{0, i}+\frac{i}{4 \alpha^{\prime}} G_{i k} \theta^{k j} \sum_{n=0}^{\infty} X_{0 n} P_{n, j} \\
Q_{1, i} \\
Q_{2, i} \\
\vdots
\end{array}\right], } \\
\left.\mid \overline{\mathcal{Q}}_{i}\right)= & {\left[\begin{array}{c}
\bar{Q}_{0, i}+\frac{i}{4 \alpha^{\prime}} G_{i k} \theta^{k j} \sum_{n=0}^{\infty} X_{0 n} \bar{P}_{n, j} \\
\bar{Q}_{1, i} \\
\bar{Q}_{2, i} \\
\vdots
\end{array}\right] . }
\end{aligned}
$$

In eqs. (4.25), passing the vectors through the phase factor of the $\left|\hat{V}_{4}\right\rangle$ and using the continuity conditions in the Neumann case

$$
\begin{aligned}
& \left.\left.(1+X) \mid P_{i}\right)\left|V_{4}\right\rangle^{X}=(1-X) \mid \bar{P}_{i}\right)\left|V_{4}\right\rangle^{X}=0 \\
& \left.\left.(1-X) \mid Q_{i}\right)\left|V_{4}\right\rangle^{X}=(1+X) \mid \bar{Q}_{i}\right)\left|V_{4}\right\rangle^{X}=0
\end{aligned}
$$

we obtain the equations, which the coefficients $Z_{n m}$ 's should satisfy,

$$
\begin{aligned}
& {\left[(1-X)_{m 0} \sum_{n=0}^{\infty}\left(\bar{Z}_{0 n}+i \frac{\pi}{2} \bar{X}_{0 n}\right) P_{n, j}+\sum_{n=1}^{\infty}(1-X)_{m n} \sum_{n^{\prime}=0}^{\infty} \bar{Z}_{n n^{\prime}} P_{n^{\prime}, j}\right]\left|V_{4}\right\rangle^{X}=0} \\
& {\left[(1+X)_{m 0} \sum_{n=0}^{\infty}\left(Z_{0 n}-i \frac{\pi}{2} X_{0 n}\right) \bar{P}_{n, j}+\sum_{n=1}^{\infty}(1+X)_{m n} \sum_{n^{\prime}=0}^{\infty} Z_{n n^{\prime}} \bar{P}_{n^{\prime}, j}\right]\left|V_{4}\right\rangle^{X}=0}
\end{aligned}
$$

for $m \geq 0$. Now all our remaing task is to solve these equations. It is easy to see that a solution of them is given by

$$
\begin{aligned}
Z_{m n} & =-i \frac{\pi}{2}(1-X)_{m n}+i \beta \frac{\pi}{2} C_{m n}, \quad(m, n \geq 0, \text { except the } m=n=0 \text { case }) \\
Z_{00} & =i \beta \frac{\pi}{2}
\end{aligned}
$$


if we pay attention to eqs. (4.28). Here $\beta$ is an unknown real constant, which is not fixed by the continuity conditions alone. This ambiguity of the solution comes from the property of the matrix $X: X C=-C X$. However it will become clear that the term containing the constant $\beta$ does not contribute to the vertex $\left|\hat{V}_{4}\right\rangle^{X}$.

Therefore, we have the expression of the phase (4.21)

$$
\theta^{i j}\left(P_{i}|Z| \bar{P}_{j}\right)=\theta^{i j}\left[i \frac{\pi}{2} P_{0, i} \bar{P}_{0, j}+i \beta \frac{\pi}{2} \sum_{n=0}^{\infty}(-1)^{n} P_{n, i} \bar{P}_{n, j}-i \frac{\pi}{2} \sum_{m, n=0}^{\infty} P_{m, i}(1-X)_{m n} \bar{P}_{n, j}\right] .
$$

Then recalling the eqs. (4.28) again, the last term in the r. h. s. can be discarded. Also we can rewrite the term containing $\beta$

$$
\begin{aligned}
-\frac{\theta^{i j}}{4 \alpha^{\prime}} \beta\left(P_{i}|C| \bar{P}_{j}\right) & =+\frac{\theta^{i j}}{4 \alpha^{\prime}} \beta\left(P_{i}\left|X^{T} C X\right| \bar{P}_{j}\right) \\
& =+\frac{\theta^{i j}}{4 \alpha^{\prime}} \beta\left(P_{i}|C| \bar{P}_{j}\right),
\end{aligned}
$$

on $\left|V_{4}\right\rangle^{X}$. When going to the second line, the properties of the matrix $X$ (A.18) were used. The above formula means that the term containing $\beta$ can be set to zero on $\left|V_{4}\right\rangle^{X}$. After all, the form of the 4-string vertex becomes

$$
\left|\hat{V}_{4}\right\rangle_{1234}^{X}=\exp \left[-\frac{\theta^{i j}}{4 \alpha^{\prime}} P_{0, i} \bar{P}_{0, j}\right]\left|V_{4}\right\rangle_{1234}^{X}
$$

Note that the phase factor has the cyclic symmetric form

$$
-\frac{\theta^{i j}}{4 \alpha^{\prime}} P_{0, i} \bar{P}_{0, j}=i \frac{\theta^{i j}}{8 \alpha^{\prime}}\left(p_{0 . i}^{(1)} p_{0, j}^{(2)}+p_{0 . i}^{(2)} p_{0, j}^{(3)}+p_{0 . i}^{(3)} p_{0, j}^{(4)}+p_{0 . i}^{(4)} p_{0, j}^{(1)}\right)
$$

which is a property the vertices should have

\section{$4.4 \quad\left|\hat{V}_{3}\right\rangle_{123}^{X}$}

We can obtain the 3-string overlap in the similar manner as in the 4-string case. First, we introduce the $Z_{3}$-Fourier transformed variables

$$
\begin{aligned}
Q_{1}^{j}(\sigma) & =\frac{1}{\sqrt{3}}\left[e X^{(1) j}(\sigma)+\bar{e} X^{(2) j}(\sigma)+X^{(3) j}(\sigma)\right] \equiv Q^{j}(\sigma), \\
Q_{2}^{j}(\sigma) & =\frac{1}{\sqrt{3}}\left[\bar{e} X^{(1) j}(\sigma)+e X^{(2) j}(\sigma)+X^{(3) j}(\sigma)\right] \equiv \bar{Q}^{j}(\sigma), \\
Q_{3}^{j}(\sigma) & =\frac{1}{\sqrt{3}}\left[X^{(1) j}(\sigma)+X^{(2) j}(\sigma)+X^{(3) j}(\sigma)\right],
\end{aligned}
$$

\footnotetext{
${ }^{8}$ Here the momentum $p_{0, i}^{(r)}$ is given by $p_{0, i}^{(r)}=\sqrt{2 \alpha^{\prime}} p_{i}^{(r)}$.
} 
where $e \equiv e^{i 2 \pi / 3}, \bar{e} \equiv e^{-i 2 \pi / 3}$. The momenta $P_{1, i}(\sigma)\left(\equiv P_{i}(\sigma)\right), P_{2, i}(\sigma)\left(\equiv \bar{P}_{i}(\sigma)\right)$ and $P_{3, i}(\sigma)$ are defined in the same way. The mode expansions take the same form as those in eqs. (4.17). In these variables, the continuity conditions require

$$
\begin{aligned}
Q^{j}(\sigma)-e Q^{j}(\pi-\sigma) & =0, & & P_{i}(\sigma)+e P_{i}(\pi-\sigma)=0, \\
\bar{Q}^{j}(\sigma)-\bar{e} \bar{Q}^{j}(\pi-\sigma) & =0, & & \bar{P}_{i}(\sigma)+\bar{e} \bar{P}_{i}(\pi-\sigma)=0, \\
Q_{3}^{j}(\sigma)-Q_{3}^{j}(\pi-\sigma) & =0, & & P_{3, i}(\sigma)+P_{3, i}(\pi-\sigma)=0
\end{aligned}
$$

for $0 \leq \sigma \leq \frac{\pi}{2}$. The conditions imposed to the modes with respect to the $t=3$ component are identical with those in the Neumann case

$$
\left.\left.(1+C) \mid P_{3, i}\right)\left|\hat{V}_{3}\right\rangle^{X}=(1-C) \mid Q_{3, i}\right)\left|\hat{V}_{3}\right\rangle^{X}=0 .
$$

Thus the $t=3$ component does not couple with $\theta^{i j}$, so we can find the solution by determining the single anti-hermitian matrix $Z$ in the phase factor whose form is assumed as

$$
\frac{1}{2} \theta^{i j} \sum_{r, s=1}^{3}\left(p_{i}^{(r)}\left|Z^{r s}\right| p_{j}^{(s)}\right)=\theta^{i j}\left(P_{i}|Z| \bar{P}_{j}\right) .
$$

For the sectors of $t=1$ and 2 , the same argument goes on as in the 4-string case. $Q^{j}(\sigma)$ and $\bar{Q}^{j}(\sigma)$ have the mode expansions same as in eqs. (4.22) and (4.23). The conditions we have to consider are

$$
\begin{aligned}
& \Delta Q^{j}(\sigma)= \begin{cases}e \Delta Q^{j}(\pi-\sigma) & \left(0 \leq \sigma \leq \frac{\pi}{2}\right) \\
\bar{e} \Delta Q^{j}(\pi-\sigma) & \left(\frac{\pi}{2} \leq \sigma \leq \pi\right),\end{cases} \\
& \Delta \bar{Q}^{j}(\sigma)= \begin{cases}\bar{e} \Delta \bar{Q}^{j}(\pi-\sigma) & \left(0 \leq \sigma \leq \frac{\pi}{2}\right) \\
e \Delta \bar{Q}^{j}(\pi-\sigma) & \left(\frac{\pi}{2} \leq \sigma \leq \pi\right),\end{cases}
\end{aligned}
$$

which are rewritten as the relations between the modes

$$
\left.\left.(1-Y) \mid \mathcal{Q}_{i}\right)\left|\hat{V}_{3}\right\rangle^{X}=\left(1-Y^{T}\right) \mid \overline{\mathcal{Q}}_{i}\right)\left|\hat{V}_{3}\right\rangle^{X}=0 .
$$

The matrix $Y$ is defined below eqs. (A.27). Recalling the conditions in the Neumann case

$$
\begin{aligned}
& \left.\left.(1+Y) \mid P_{i}\right)\left|V_{3}\right\rangle^{X}=\left(1+Y^{T}\right) \mid \bar{P}_{i}\right)\left|V_{3}\right\rangle^{X}=0 \\
& \left.\left.(1-Y) \mid Q_{i}\right)\left|V_{3}\right\rangle^{X}=\left(1-Y^{T}\right) \mid \bar{Q}_{i}\right)\left|V_{3}\right\rangle^{X}=0
\end{aligned}
$$

we end up with the following equations

$$
\begin{aligned}
& {\left[(1-Y)_{m 0} \sum_{n=0}^{\infty}\left(\bar{Z}_{0 n}+\frac{\pi}{2} \bar{X}_{0 n}\right) P_{n, j}+\sum_{n=1}^{\infty}(1-Y)_{m n} \sum_{n^{\prime}=0}^{\infty} \bar{Z}_{n n^{\prime}} P_{n^{\prime}, j}\right]\left|V_{3}\right\rangle^{X}=0} \\
& {\left[\left(1-Y^{T}\right)_{m 0} \sum_{n=0}^{\infty}\left(Z_{0 n}-i \frac{\pi}{2} X_{0 n}\right) \bar{P}_{n, j}+\sum_{n=1}^{\infty}\left(1-Y^{T}\right)_{m n} \sum_{n^{\prime}=0}^{\infty} Z_{n n^{\prime}} \bar{P}_{n^{\prime}, j}\right]\left|V_{3}\right\rangle^{X}=0(4 .}
\end{aligned}
$$


for $m \geq 0$. It can be easily found out that the expression

$$
\begin{aligned}
Z_{m n} & =-i \frac{\pi}{\sqrt{3}}\left(1+Y^{T}\right)_{m n} \quad(m, n \geq 0, \text { except the } m=n=0 \text { case }), \\
Z_{00} & =0
\end{aligned}
$$

satisfies the above equations. It should be noted that in this case, because of $C Y C=\bar{Y} \neq$ $-Y$, it does not contain any unknown constant differently from the 4 -string case.

Owing to the condition (4.43) we can write the phase factor only in terms of the zeromodes. Finally we have

$$
\begin{aligned}
\left|\hat{V}_{3}\right\rangle_{123}^{X} & =\exp \left[-\frac{\theta^{i j}}{4 \sqrt{3} \alpha^{\prime}} P_{0, i} \bar{P}_{0, j}\right]\left|V_{3}\right\rangle_{123}^{X} \\
& =\exp \left[i \frac{\theta^{i j}}{12 \alpha^{\prime}}\left(p_{0, i}^{(1)} p_{0, j}^{(2)}+p_{0, i}^{(2)} p_{0, j}^{(3)}+p_{0, i}^{(3)} p_{0, j}^{(1)}\right)\right]\left|V_{3}\right\rangle_{123}^{X} .
\end{aligned}
$$

It is not clear whether the solutions we have obtained here are unique or not. However we can show that the phase factors are consistent with the relations between the overlaps which they should satisfy :

$$
{ }_{3}\left\langle\hat{I} \mid \hat{V}_{3}\right\rangle_{123}=\left|\hat{V}_{2}\right\rangle_{12}, \quad{ }_{4}\left\langle\hat{I} \mid \hat{V}_{4}\right\rangle_{1234}=\left|\hat{V}_{3}\right\rangle_{123}, \quad{ }_{34}\left\langle\hat{V}_{2}|| \hat{V}_{3}\right\rangle_{123}\left|\hat{V}_{3}\right\rangle_{456}=\left|\hat{V}_{4}\right\rangle_{1256},
$$

by using the momentum conservation on the vertices $\left(p_{i}^{(1)}+\cdots+p_{i}^{(N)}\right)\left|\hat{V}_{N}\right\rangle_{1 \cdots N}^{X}=0$. Furthermore we can see that the phase factors successfully reproduce the Moyal product structures of the correlators among vertex operators obtained in the perturbative approach to open string theory in the constant $B$-field background [10]. These facts convince us that the solutions obtained here are physically meaningful.

\section{Transformation of String Fields}

In the previous section, we have explicitly constructed the overlap vertices in the operator formulation under the constant $B$-field background. Then we have obtained the vertices with a new noncommutative structure of the Moyal type originating from the constant $B$-field, in addition to the ordinary product $*$ of string fields. Denoting the product with the new structure by $\star$, the action of the string field theory is written as

$$
\begin{aligned}
S_{B} & =\frac{1}{G_{s}} \int\left(\frac{1}{2} \psi \star Q \psi+\frac{1}{3} \psi \star \psi \star \psi\right) \\
& =\frac{1}{G_{s}}\left(\frac{1}{2}{ }_{12}\left\langle\hat{V}_{2}|| \psi\right\rangle_{1} Q|\psi\rangle_{2}+\frac{1}{3}{ }_{123}\left\langle\hat{V}_{3}|| \psi\right\rangle_{1}|\psi\rangle_{2}|\psi\rangle_{3}\right),
\end{aligned}
$$

where the BRST charge $Q$ is constructed from the world sheet action (3.1). The theory (5.1) gives the noncommutative $U(1)$ Yang-Mills theory in the low energy region in the same sense 
as Witten's open string field theory in the case of the Neumann boundary condition leads to the ordinary $U(1)$ Yang-Mills theory in the low energy limitl?

In ref. [10], Seiberg and Witten argued that open string theory in the constant $B$-field background leads to either commutative or noncommutative Yang-Mills theories, corresponding to the different regularization scheme - the Pauli-Villars regularization or the pointsplitting regularization - in the world sheet formulation. They discussed a map between the gauge fields in the commutative and noncommutative Yang-Mills theories. In string field theory perspective, there also should be a certain transformation (hopefully simpler than the Yang-Mills case) from the string field $\psi$ in (5.1) to a string field in a new string field theory which leads to the commutative Yang-Mills theory in the low energy limit.

Here we obtain the new string field theory by finding a unitary transformation which absorbs the noncommutative structure of the Moyal type in the product $\star$ into a redefinition of the string fields. There are used the two vertices $\left|\hat{V}_{2}\right\rangle$ and $\left|\hat{V}_{3}\right\rangle$ in the action (5.1). Recall that the 2-string vertex is in the same form as in the Neumann case and has no Moyal type noncommutative structure. First, we consider the phase factor of the 3-string vertex which multiplies in front of $\left|V_{3}\right\rangle$ (See eq. (4.48).). Making use of the continuity conditions

$$
P_{0, i}=-2 \sum_{n=1}^{\infty} Y_{0 n} P_{n, i}, \quad \bar{P}_{0, i}=-2 \sum_{n=1}^{\infty} \bar{Y}_{0 n} \bar{P}_{n, i}
$$

it can be rewritten as

$$
\begin{aligned}
-\frac{\theta^{i j}}{4 \sqrt{3} \alpha^{\prime}} P_{0, i} \bar{P}_{0, j} & =\frac{\theta^{i j}}{4 \sqrt{3} \alpha^{\prime}} \sum_{n=1}^{\infty}\left(P_{0, i} \bar{Y}_{0 n} \bar{P}_{n, j}+P_{n, i} Y_{0 n} \bar{P}_{0, j}\right) \\
& =-\frac{\theta^{i j}}{24 \alpha^{\prime}} \sum_{n=1}^{\infty} X_{0 n}\left[\left(-p_{0, i}^{(2)}-p_{0, i}^{(3)}+2 p_{0, i}^{(1)}\right) p_{n, j}^{(1)}\right. \\
& \left.\quad+\left(-p_{0, i}^{(3)}-p_{0, i}^{(1)}+2 p_{0, i}^{(2)}\right) p_{n, j}^{(2)}+\left(-p_{0, i}^{(1)}-p_{0, i}^{(2)}+2 p_{0, i}^{(3)}\right) p_{n, j}^{(3)}\right] \\
& =-\frac{\theta^{i j}}{8 \alpha^{\prime}} \sum_{r=1}^{3} \sum_{n=1}^{\infty} X_{0 n} p_{0, i}^{(r)} p_{n, j}^{(r)},
\end{aligned}
$$

where we used the property of the matrix $Y: Y_{0 n}=-\bar{Y}_{0 n}=\frac{\sqrt{3}}{2} X_{0 n}$ for $n \geq 1$ and the momentum conservation on $\left|V_{3}\right\rangle: p_{0, i}^{(1)}+p_{0, i}^{(2)}+p_{0, i}^{(3)}=0$. We manage to represent the phase factor of the Moyal type as a form factorized into the product of the unitary operators

$$
\mathcal{U}_{r}=\exp \left(\frac{\theta^{i j}}{8 \alpha^{\prime}} \sum_{n=1,3,5, \cdots} X_{0 n} p_{0, i}^{(r)} p_{n, j}^{(r)}\right) .
$$

Note that the unitary operator acts to a single string field. So the Moyal type noncommutativity can be absorbed by the unitary rotation of the string field

$$
\begin{aligned}
{ }_{123}\left\langle\hat{V}_{3}|| \psi\right\rangle_{1}|\psi\rangle_{2}|\psi\rangle_{3} & ={ }_{123}\left\langle V_{3}\left|\mathcal{U}_{1} \mathcal{U}_{2} \mathcal{U}_{3}\right| \psi\right\rangle_{1}|\psi\rangle_{2}|\psi\rangle_{3} \\
& ={ }_{123}\left\langle V_{3}|| \tilde{\psi}\right\rangle_{1}|\tilde{\psi}\rangle_{2}|\tilde{\psi}\rangle_{3}
\end{aligned}
$$

\footnotetext{
${ }^{9}$ It can be explicitly seen by repeating a similar calculation as that carried out in ref. 21.
} 
with $|\tilde{\psi}\rangle_{r}=\mathcal{U}_{r}|\psi\rangle_{r}$. It should be remarked that this manipulation has been suceeded owing to the factorized expression of the phase factor, which originates from the continuity conditions relating the zero-modes to the nonzero-modes (5.2). It is a characteristic feature of string field theory that can not be found in any local field theories.

Next let us see the kinetic term. In doing so, it is judicious to write the kinetic term as follows:

$$
{ }_{12}\left\langle\hat{V}_{2}|| \psi\right\rangle_{1}\left(Q|\psi\rangle_{2}\right)={ }_{123}\left\langle\hat{V}_{3}|| \psi\right\rangle_{1}\left(Q_{L}|I\rangle_{2}|\psi\rangle_{3}+|\psi\rangle_{2} Q_{L}|I\rangle_{3}\right),
$$

where $Q_{L}$ is defined by integrating the BRST current $j_{B R S T}(\sigma)$ with respect to $\sigma$ over the left half region

$$
Q_{L}=\int_{0}^{\pi / 2} d \sigma j_{B R S T}(\sigma)
$$

Eq. (5.6) is also represented by the product $\star$ as

$$
\psi \star(Q \psi)=\psi \star\left[\left(Q_{L} I\right) \star \psi+\psi \star\left(Q_{L} I\right)\right] .
$$

$I$ stands for the identity element with respect to the $\star$-product, carrying the ghost number $-\frac{3}{2}$, which corresponds to $|I\rangle$ in the operator formulation. As is discussed in ref. [19], in order to show the relation (5.8) we need the formulas

$$
\begin{aligned}
& Q_{R} I=-Q_{L} I, \\
& \left(Q_{R} \psi\right) \star \xi=-(-1)^{n_{\psi}} \psi \star\left(Q_{L} \xi\right)
\end{aligned}
$$

for arbitrary string fields $\psi$ and $\xi$, where $Q_{R}$ is the integrated BRST current over the right half region of $\sigma \cdot n_{\psi}$ stands for the ghost number of the string field $\psi$ minus $\frac{1}{2}$, and takes an integer value. The first formula means that the identity element is a physical quantity, also the second does the conservation of the BRST charget term in the bracket in r. h. s. of eq. (5.8) becomes

$$
\left(Q_{L} I\right) \star \psi=-\left(Q_{R} I\right) \star \psi=I \star\left(Q_{L} \psi\right)=Q_{L} \psi
$$

Also, it turns out that the second term is equal to $Q_{R} \psi$. Combining these, we can see that the eq. (5.8) holds.

Further, we should remark that because the BRST current does not contain the center of mass coordinate $x^{j}$, it commute with the momentum $p_{i}$. From the continuity condition $p_{i}|I\rangle=0$, it can be seen that $p_{i} Q_{L}|I\rangle=0$. Expanding the exponential in the expression of the unitary operator (5.4) and passing the momentum $p_{0, i}$ to the right, we obtain

$$
\mathcal{U} Q_{L}|I\rangle=Q_{L}|I\rangle
$$

\footnotetext{
${ }^{10}$ For a proof of these formulas, we can use the same argument as in ref. 19 and references therein.
} 
Now we can write down the result of the kinetic term. As a result of the same manipulation as in eq. (5.5) and the use of eq. (5.10), we have"

$$
\begin{aligned}
{ }_{12}\left\langle\hat{V}_{2} \| \psi\right\rangle_{1}\left(Q|\psi\rangle_{2}\right) & ={ }_{123}\left\langle\hat{V}_{3}|| \psi\right\rangle_{1}\left(Q_{L}|I\rangle_{2}|\psi\rangle_{3}+|\psi\rangle_{2} Q_{L}|I\rangle_{3}\right) \\
& ={ }_{123}\left\langle V_{3}|| \tilde{\psi}\right\rangle_{1}\left(Q_{L}|I\rangle_{2}|\tilde{\psi}\rangle_{3}+|\tilde{\psi}\rangle_{2} Q_{L}|I\rangle_{3}\right) \\
& ={ }_{12}\left\langle V_{2}|| \tilde{\psi}\right\rangle_{1}\left(Q|\tilde{\psi}\rangle_{2}\right) .
\end{aligned}
$$

Here we have a comment. If we considered the kinetic term itself without using eq. (5.6), what would be going on? Let us see this. From the continuity conditions for $\left|\hat{V}_{2}\right\rangle_{12}^{X}=\left|V_{2}\right\rangle_{12}^{X}$ :

$$
p_{0, i}^{(1)}+p_{0, i}^{(2)}=0, \quad p_{n, i}^{(1)}+(-1)^{n} p_{n, i}^{(2)}=0 \quad(n=1,2, \cdots),
$$

it could be shown that the 2-string overlap is invariant under the unitary rotation

$$
\mathcal{U}_{1} \mathcal{U}_{2}\left|V_{2}\right\rangle_{12}=\left|V_{2}\right\rangle_{12}
$$

So we would find the expression for the kinetic term after the rotation

$$
{ }_{12}\left\langle V_{2}|| \psi\right\rangle_{1} Q|\psi\rangle_{2}={ }_{12}\left\langle V_{2}|| \tilde{\psi}\right\rangle_{1} \tilde{Q}|\tilde{\psi}\rangle_{2}
$$

where $\tilde{Q}$ is the BRST charge similarity transformed by $\mathcal{U}$

$$
\tilde{Q}=\mathcal{U} Q \mathcal{U}^{\dagger}
$$

However, after some computations of the r. h. s. of eq. (5.15), we could see that $\tilde{Q}$ has divergent term proportional to

$$
\sum_{n=1,3,5, \cdots} 1
$$

and thus it is not well-defined. It seems that this procedure is not correct and needs some suitable regularization, which preserves the conformal symmetry ${ }^{[2}$. It is considered that the

\footnotetext{
${ }^{11}$ Strictly speaking, in general this formula holds in the case that both of the string fields $|\psi\rangle$ and $|\tilde{\psi}\rangle$ belong to the Fock space which consists of states excited by finite number of creation operators. This point is subtle for giving a proof. However, for the infinitesimal $\theta$ case, by keeping arbitrary finite order terms in the expanded form of the exponential of $\mathcal{U}$, we can make the situation of both $|\psi\rangle$ and $|\tilde{\psi}\rangle$ being inside the Fock space, and thus clearly eq. (5.11) holds. From this fact, it is plausible to expect that eq. (5.11) is correct in the finite $\theta$ case.

${ }^{12}$ That divergence comes from the mid-point sigularity of the string coordinates transformed by $\mathcal{U}$. In fact, after some calculations, we have

$$
\mathcal{U} X^{j}(\sigma) \mathcal{U}^{\dagger}=X^{j}(\sigma)-i \frac{\theta^{j k}}{4 \sqrt{2 \alpha^{\prime}}} \sum_{n=1,3,5, \cdots} X_{n 0} p_{n, k}-\frac{\theta^{j k}}{4} p_{k} \operatorname{sgn}\left(\sigma-\frac{\pi}{2}\right) .
$$

The last term leads to the mid-point sigularity in the energy-momentum tensor and the BRST charge $Q$. It seems that the use of eq. (5.6) corresponds to taking the point splitting regularization with respect to the mid-point. Because of the discontinuity of the last term in eq. (5.16), it is considered that the transformed string coordinates have no longer a good picture as a string. It could be understood from the point that the transformation $\mathcal{U}$ drives states around a perturbative vacuum to those around highly nonperturbative one like coherent states.
} 
use of eq. (5.6) gives that kind of regularization, which will be justified at the end of the next section.

Therefore the string field theory action (5.1) with the Moyal type noncommutativity added to the ordinary noncommutativity is equivalently rewritten as the one with the ordinary noncommutativity alone:

$$
\begin{aligned}
S_{B} & =\frac{1}{G_{s}} \int\left(\frac{1}{2} \tilde{\psi} * Q \tilde{\psi}+\frac{1}{3} \tilde{\psi} * \tilde{\psi} * \tilde{\psi}\right) \\
& =\frac{1}{G_{s}}\left(\frac{1}{2}{ }_{12}\left\langle V_{2}|| \tilde{\psi}\right\rangle_{1} Q|\tilde{\psi}\rangle_{2}+\frac{1}{3}{ }_{123}\left\langle V_{3}|| \tilde{\psi}\right\rangle_{1}|\tilde{\psi}\rangle_{2}|\tilde{\psi}\rangle_{3}\right) .
\end{aligned}
$$

It is noted that the BRST charge $Q$, which is constructed from the world sheet action (3.1), has the same form as the one obtained from the action (2.5) with the relation (3.7). So all the $B$-dependence has been stuffed into the string fields except that existing in the metric $G_{i j}$. Furthermore, recalling that the relation between the metrics $G^{i j}$ and $g_{i j}$ is the same form as the T-duality inversion transformation, which was pointed out at the end of section 3 , we can make the metric $g_{i j}$ appear in the overlap vertices, instead of the metric $G_{i j}$. To do so, we consider the following transformation for the modes:

$$
\hat{\alpha}_{n}^{i}=\left(E^{T-1}\right)^{i k} \alpha_{n, k}, \quad \hat{p}^{i}=\left(E^{T-1}\right)^{i k} p_{k}, \quad \hat{x}_{i}=E_{i k} x^{k} .
$$

By this transformation, the commutators become

$$
\left[\hat{\alpha}_{n}^{i}, \hat{\alpha}_{m}^{j}\right]=n g^{i j} \delta_{n+m, 0}, \quad\left[\hat{p}^{i}, \hat{x}_{j}\right]=-i \delta_{j}^{i},
$$

and the bilinear form of the modes

$$
\begin{aligned}
G^{i j} \alpha_{n, i} \alpha_{m, j} & =g_{i j} \hat{\alpha}_{n}^{i} \hat{\alpha}_{m}^{j}, \\
G^{i j} p_{i} \alpha_{m, j} & =g_{i j} \hat{p}^{i} \hat{\alpha}_{m}^{j}, \\
G^{i j} p_{i} p_{j} & =g_{i j} \hat{p}^{i} \hat{p}^{j} .
\end{aligned}
$$

Since in viewing the expressions of the overlaps (A.6) and (A.25) the metric $G_{i j}$ appears only through the bilinear forms (5.20), now the dependence of the background $B_{i j}$ can be completely removed from the overlaps $\left|V_{2}\right\rangle$ and $\left|V_{3}\right\rangle$ in the action (5.17) after the transformation (5.18).

Remark that we have performed the unitary rotation and the T-duality inversion but have not any shift of the string field. In string field theory perspective, the background is changed by shifting the string field by a solution of the classical string field equation. Because we have done no such a shift, it is possible to consider that the two actions (5.1) and (5.17) describe open strings in the same background. The one (5.1) certainly leads to the noncommutative Yang-Mills theory in the low energy limit. Also, it is clear that the other (5.17) gives the commutative Yang-Mills theory as the low energy effective theory. Thus it is considered that the unitary transformation $|\tilde{\psi}\rangle=\mathcal{U}|\psi\rangle$ combined with the T-duality inversion (5.18) corresponds to a microscopic analog of the map between the gauge fields in the commutative and noncommutative Yang-Mills theories in ref. [10]. 
We end this section by noting a following comment. We have had the string field theories which describe the commutaive and noncommutative Yang-Mills theories at the low energy region. They have the identical string coupling $G_{s}$. On the other hand, according to an analysis of the Dirac-Born-Infeld (DBI) actions corresponding to the commutative and noncommutative Yang-Mills theories in ref. [10], it turns out that the coupling constants of the

commutative and noncommutative DBI actions, denoted by $g_{s}^{(D B I)}$ and $G_{s}^{(D B I)}$ respectively, differ by a factor depending on the metrics:

$$
G_{s}^{(D B I)}(\operatorname{det} G)^{-1 / 4}=g_{s}^{(D B I)}(\operatorname{det} g)^{-1 / 4} .
$$

This relation seems to be nontrivial from our point of view. In the commutative theory we have had, since all the $B$-dependence is contained in infinite number of wave functions in the string fields, it seems to need more consideration to derive eq. (5.21). We will discuss this issue elsewhere.

\section{Background Independence}

In this section, we show that both theories in the background described by the world sheet action (3.1) and in the background of the metric $g_{i j}$ alone are derived from the single purely cubic action

$$
S_{3}=\frac{1}{3 G_{s}} \int \psi \star \psi \star \psi=\frac{1}{3 G_{s}} 123\left\langle\hat{V}_{3}|| \psi\right\rangle_{1}|\psi\rangle_{2}|\psi\rangle_{3},
$$

by using the unitary transformation (5.4) together with an argument in ref. [19]. The result gives a new type of the background independence connecting between theories with different boundary conditions caused by the background $B_{i j}$.

First we note that the cubic action has a universal structure. The Moyal type noncommutativity is absorbed into the redefinition of the string field $|\tilde{\psi}\rangle=\mathcal{U}|\psi\rangle$ as discussed in the previous section. Also we can get rid of the dependence of the metric $G_{i j}$. Since $G_{i j}$ can be diagonalized by an appropriate orthogonal matrix, we rotate the modes $\alpha_{n, i}, p_{i}$ and $x^{i}$ by the orthogonal matrix and absorb the eigenvalues into suitable rescalings of the modes. So the metric appearing in the commutators and in the bilinear forms can be made Minkowskian:

$$
\begin{aligned}
{\left[\alpha_{n, i}, \alpha_{m, j}\right]=n G_{i j} \delta_{n+m, 0} } & \Rightarrow\left[\alpha_{n, i}, \alpha_{m, j}\right]=n \eta_{i j} \delta_{n+m, 0}, \\
G^{i j} \alpha_{n, i} \alpha_{m, j} & \Rightarrow \eta^{i j} \alpha_{n, i} \alpha_{m, j}, \\
G^{i j} p_{i} \alpha_{m, j} & \Rightarrow \eta^{i j} p_{i} \alpha_{m, j} \\
G^{i j} p_{i} p_{j} & \Rightarrow \eta^{i j} p_{i} p_{j} .
\end{aligned}
$$

As a result, the cubic action (6.1) is to have no specific dependence of the background $g_{i j}$ and $B_{i j}$. It shows that the universality of the interaction vertices in string field theory discussed in refs. [17, 18, 19] holds also between the open string field theories with different boundary conditions. 
The classical equation of motion obtained from the action (6.1) is

$$
\psi \star \psi=0
$$

Let us denote the BRST charge derived from the world sheet action (3.1) by $Q$. We see that $\psi=Q_{L} I$ gives a solution of (6.3) by using the properties

$$
\left\{Q, Q_{L}\right\}=0
$$

in addition to eqs. (5.9). When showing eq. (6.4) by a direct calculation, it is crucial that the BRST current $j_{B R S T}$ is a primary field with the conformal weight 1 . We decompose the string field $\psi$ into the solution $Q_{L} I$ and the fluctuation around it $\Psi$. Then, we can rewrite the cubic action into Witten's form (5.1) by using eq. (5.6).

On the other hand, via the unitary transformation (5.4), the cubic action (6.1) becomes

$$
S_{3}=\frac{1}{3 G_{s}} \int \tilde{\psi} * \tilde{\psi} * \tilde{\psi}=\frac{1}{3 G_{s}}{ }_{123}\left\langle V_{3}|| \tilde{\psi}\right\rangle_{1}|\tilde{\psi}\rangle_{2}|\tilde{\psi}\rangle_{3} .
$$

We write as $Q^{0}$ the BRST charge corresponding to the world sheet action in the background of the metric $g_{i j}$ alone. After considering the transformation (5.18), it is found that $\tilde{\psi}=Q_{L}^{0} I$ satisfies the equation of motion $\tilde{\psi} * \tilde{\psi}=0$. We expand the string field around this solution as $\tilde{\psi}=Q_{L}^{0} I+\tilde{\Psi}$, and then the string field action in the background of the metric alone is obtained:

$$
S^{0}=\frac{1}{G_{s}} \int\left(\frac{1}{2} \tilde{\Psi} *\left(Q^{0} \tilde{\Psi}\right)+\frac{1}{3} \tilde{\Psi} * \tilde{\Psi} * \tilde{\Psi}\right) .
$$

By combining these two facts we conclude that the theory in the background $g_{i j}$ and $B_{i j}$ (5.1) is connected to the theory in the background $g_{i j}$ alone (6.6). It is the advertised result.

At the end of this section we give a justification of the regularization used in the previous

section by showing the equivalence of the actions (5.1) and (5.17). If we do not consider the transformation (5.18) in the action (6.5), we naturally find $\tilde{\psi}=Q_{L} I$ as a solution. In fact, because both BRST currents corresponding to $Q^{0}$ and $Q$ are primary fields with the conformal weight 1 , they are qualified to be a background of string theory. After the same procedure as the above, we have the action

$$
S=\frac{1}{G_{s}} \int\left(\frac{1}{2} \tilde{\Psi} *(Q \tilde{\Psi})+\frac{1}{3} \tilde{\Psi} * \tilde{\Psi} * \tilde{\Psi}\right),
$$

which represents the theory in the background $g_{i j}$ and $B_{i j}$. Thus it turns out that the actions (5.1) and (5.7) describe the theory in the same background, which is nothing but the equivalence of eq. (5.1) and eq. (5.17).

\section{Discussions}

We have considered Witten's open string field theory in the presence of a constant background of the second-rank antisymmetric tensor field $B_{i j}$. We have extended the operator 
formulation based on a Fock space representation of string fields and overlap vertices in this situation, and have constructed explicitly the overlap vertices.

As a result, we have found a new kind of noncommutative structure in addition to the ordinary noncommutativity which exists irrespective of the $B$-field background. It appears only in a part of the string field product, which is the part concerning the zero-modes, and the form is a product of the Moyal type. It is consistent with the result of the correlation functions among vertex operators in the world sheet formulation. The string field theory obtained here gives the noncommutative Yang-Mills theory in the low energy limit.

Furthermore we have found that the Moyal type noncommutativity can be absorbed into a redefinition of the string fields by a unitary transformation. After the transformation, the string field theory is rewritten into a string field theory with the ordinary noncommutative product. Although there still exists the $B$-field dependence in the metric appearing in the overlaps, we can perform the T-duality inversion transformation so as to completely absorb it. This theory describes the commutative Yang-Mills theory in the low energy region. The unitary transformation plus the T-duality inversion transformation is considered to be a microscopic analog of the map between the gauge fields in the commutative and noncommutative Yang-Mills theories discussed in ref. [10].

Also we have considered the background independence in this situation along the same line as discussed in ref. [19]. It has been shown that the theory in the background of the $B$-field as well as the metric is connected to that in the background of the metric alone by an appropriate redefinition of the string fields. We have seen that the universality of the interaction vertices in string field theory argued before in refs. [17, 18, 19] holds also between the open string theories with different boundary conditons caused by the $B$-field background.

Until now, we have not put the Chan-Paton factors to the end points of open strings, which corresponds to considering the case of the $U(1)$-gauge group in Yang-Mills theory. However, as is pointed out in refs. [20, 21], it is a straghtforward generalization to introduce the Chan-Paton factors in Witten's open string field theory:

$$
\begin{aligned}
S^{C P} & =\frac{1}{G_{s}} \int\left(\frac{1}{2} \psi_{a} * Q \psi_{b} \operatorname{tr}\left(\lambda_{a} \lambda_{b}\right)+\frac{1}{3} \psi_{a} * \psi_{b} * \psi_{c} \operatorname{tr}\left(\lambda_{a} \lambda_{b} \lambda_{c}\right)\right) \\
& =\frac{1}{G_{s}}\left(\frac{1}{2}{ }_{12}\left\langle V_{2}|| \psi_{a}\right\rangle_{1} Q\left|\psi_{b}\right\rangle_{2} \operatorname{tr}\left(\lambda_{a} \lambda_{b}\right)+\frac{1}{3}{ }_{123}\left\langle V_{3}|| \psi_{a}\right\rangle_{1}\left|\psi_{b}\right\rangle_{2}\left|\psi_{c}\right\rangle_{3} \operatorname{tr}\left(\lambda_{a} \lambda_{b} \lambda_{c}\right)\right),
\end{aligned}
$$

where $a, b$ and $c$ stand for the Chan-Paton factors, and $\lambda_{a}$ is a generator of the gauge group. Since the structure of the gauge group and that of the overlaps appear in the factorized form, we can consider them independently. Thus we can immediately write down the string field theory action with the Chan-Paton factors in the presence of the $B$-field background as

$$
\begin{aligned}
S_{B}^{C P} & =\frac{1}{G_{s}} \int\left(\frac{1}{2} \psi_{a} \star Q \psi_{b} \operatorname{tr}\left(\lambda_{a} \lambda_{b}\right)+\frac{1}{3} \psi_{a} \star \psi_{b} \star \psi_{c} \operatorname{tr}\left(\lambda_{a} \lambda_{b} \lambda_{c}\right)\right) \\
& =\frac{1}{G_{s}}\left(\frac{1}{2}{ }_{12}\left(\hat{V}_{2}|| \psi_{a}\right\rangle_{1} Q\left|\psi_{b}\right\rangle_{2} \operatorname{tr}\left(\lambda_{a} \lambda_{b}\right)+\frac{1}{3}{ }_{123}\left\langle\hat{V}_{3}|| \psi_{a}\right\rangle_{1}\left|\psi_{b}\right\rangle_{2}\left|\psi_{c}\right\rangle_{3} \operatorname{tr}\left(\lambda_{a} \lambda_{b} \lambda_{c}\right)\right)
\end{aligned}
$$


where the product $\star$ and the overlap vertices $\left|\hat{V}_{2}\right\rangle$ and $\left|\hat{V}_{3}\right\rangle$ are identical with those constructed in the $U(1)$ case. It is clear that, by using the same argument as in the $U(1)$ case, we can absorb the Moyal type noncommutativity and obtain the string field theory leading to the commutative Yang-Mills theory in the low energy limit.

At first sight, it seems that in the form of the string field action (7.2) the noncommutativity originating from the $B$-field background (the product $\star$ ) does not have any connection with that from the nonabelian gauge group (the Chan-Paton factor). On the other hand, it is known that these two noncommutative natures are related to each other in the interesting way: "Infinitely many coincident $\mathrm{D} p$-branes can be described by a single $\mathrm{D}(p+2)$-brane in the background of some constant $B$-field." This phenomenon has been discussed in the world sheet formulation of open string theory in ref. [8, 9]. So in the framework of Witten's open string field theory constructed here, it might be interesting to examine how the noncommutativity from the nonabelian gauge group transmutes into that from the constant $B$-field background. Because in Matrix theory [23, 24 higher dimensional D-branes are constructed from the constituent D-particles by utilizing the above fact ${ }^{\mathrm{T} 3}$, it might be useful for clarifying the connection between Matrix theory and open string field theory, and further it might give a hint for considering the covariant and nonperturbative formulation of M-theory.

\section{Acknowledgements}

The author would like to express his gratitude to T. Kuroki, Y. Okawa and T. Yoneya for a lot of variable discussions about Witten's open string theory and its operator formulation for the first half of the year 1997. A part of this work was done during his visit at C. E. A. Saclay. The author would like to thank members of C. E. A. Saclay for their warm hospitality, especially I. Kostov for his kindness. Also the author wishes to thank N. Ishibashi and T. Yoneya for helpful discussions. The research of the author is supported by the Japan Society for the Promotion of Science under the Postdoctral Research Program.

\footnotetext{
${ }^{13} \mathrm{~A}$ similar phenomenon as the above is known also in the context of the IIB matrix model [25]. Noncommutative Yang-Mills theory in four dimensions arises in expanding matrices around a classical solution which represents a condensation of D-instantons and composes a D3-brane [26].
} 


\section{Appendix}

\section{A Explicit Form of Overlap Vertices in the Neumann Boundary Condition}

Here we give the explicit form of the overlap vertices $\left|V_{N}\right\rangle$, which satisfy the continuity conditions (2.11) and (2.12), in the case of $N=1,2,3,4$. Although it is a slight generalization of the result in the background of the flat space-time by Gross and Jevicki [14, 15], we present it in order to make this paper more self-contained.

\section{A.1 $|I\rangle \equiv\left|V_{1}\right\rangle$}

The overlap in the $N=1$ case behaves as an identity element for the $*$-product, so we denote it by $|I\rangle$. Its explicit form is as follows.

$$
\begin{array}{r}
|I\rangle^{X} \equiv\left|V_{1}\right\rangle^{X}=\exp \left[-\frac{1}{2} G^{i j} \sum_{n=0}^{\infty}(-1)^{n} a_{n, i}^{\dagger} a_{n, j}^{\dagger}\right]|0\rangle \\
|I\rangle^{g h} \equiv\left|V_{1}\right\rangle^{g h}=\exp \left[\sum_{n=2}^{\infty}(-1)^{n} c_{-n} b_{-n}-2 c_{0} \sum_{k=1}^{\infty}(-1)^{k} b_{-2 k}\right. \\
\left.+\left(c_{-1}-c_{1}\right) \sum_{k=1}^{\infty}(-1)^{k} b_{-2 k-1}\right]|\Omega\rangle .
\end{array}
$$

Here, the operators $a_{n, i}$ and $a_{n, i}^{\dagger}$ are given by

$$
\begin{aligned}
& \alpha_{n, i}=\sqrt{n} a_{n, i}, \quad \alpha_{-n, i}=\sqrt{n} a_{n, i}^{\dagger} \quad(n \geq 1), \\
& x_{i}=\frac{i}{2} \sqrt{2 \alpha^{\prime}}\left(a_{0, i}-a_{0, i}^{\dagger}\right), \quad p_{i}=\frac{1}{\sqrt{2 \alpha^{\prime}}}\left(a_{0, i}+a_{0, i}^{\dagger}\right),
\end{aligned}
$$

the vacuum $|0\rangle$ is annihilated by all the $a_{n, i}$ 's $(n \geq 0)$. Also $|\Omega\rangle$ in the ghost sector is the so-called $S L(2, \mathbf{R})$ vacuum satisfying

$$
\begin{aligned}
& b_{n}|\Omega\rangle=0 \quad(n \geq-1), \\
& c_{n}|\Omega\rangle=0 \quad(n \geq 2) \text {. }
\end{aligned}
$$

Note that we usually use the vacua $| \pm\rangle$ satisfying

$$
\begin{array}{ll}
b_{n}| \pm\rangle=c_{n}| \pm\rangle=0 & (n \geq 1), \\
|+\rangle=c_{0}|-\rangle, & |-\rangle=b_{0}|+\rangle .
\end{array}
$$

The vacua $|+\rangle$ and $|-\rangle$ have the ghost number $\frac{1}{2}$ and $-\frac{1}{2}$ respectively. Note that the $S L(2, \mathbf{R})$ vacuum is related to the $|+\rangle$ vacuum as $|\Omega\rangle=b_{-1} b_{0}|+\rangle$, and that it has the ghost number $-\frac{3}{2}$ which is the correct ghost number the identity overlap should have. 


\section{A.2 $\left|V_{2}\right\rangle_{12}$}

We write the 2-string overlap for two strings denoted by 1 and 2 .

$$
\begin{aligned}
\left|V_{2}\right\rangle_{12}^{X} & =\exp \left[-G^{i j} \sum_{n=0}^{\infty}(-1)^{n} a_{n, i}^{(1) \dagger} a_{n, j}^{(2) \dagger}\right]|0\rangle_{12}, \\
\left|V_{2}\right\rangle_{12}^{g h} & =\exp \left[\sum_{n=1}^{\infty}(-1)^{n}\left(c_{-n}^{(1)} b_{-n}^{(2)}+c_{-n}^{(2)} b_{-n}^{(1)}\right)\right]|\Omega\rangle_{12},
\end{aligned}
$$

where the superscripts (1) and (2) label the strings, the vacuum in the ghost sector $|\Omega\rangle_{12}$ is defined by

$$
|\Omega\rangle_{12}=\frac{1}{\sqrt{2}}\left(|-,+\rangle_{1,2}-|+,-\rangle_{1,2}\right) .
$$

When considering the connection to the string perturbation theory, it is convenient to take the momentum representation rather than the oscillator representation with respect to the zero-modes. In order to translate into the momentum representation, we need the momentum eigenstate constructed from the vacuum of the zero-mode oscillators, which is given by

$$
|p\rangle^{(0)}=\frac{1}{(\operatorname{det} G)^{1 / 4}} \exp G^{i j}\left[-\frac{1}{4} \cdot 2 \alpha^{\prime} p_{i} p_{j}+\sqrt{2 \alpha^{\prime}} p_{i} a_{0, j}^{\dagger}-\frac{1}{2} a_{0, i}^{\dagger} a_{0, j}^{\dagger}\right]|0\rangle^{(0)},
$$

where the states of the zero-mode sector were shown by the states with the superscript $(0)$. Using eq. (A.9), we can obtain the overlap in the momentum representation with respect to the zero-modes:

$$
\begin{aligned}
{ }_{1}^{(0)}\left\langle p^{(1)}\right|{ }_{2}^{(0)}\left\langle p^{(2)}|| V_{2}\right\rangle_{12}^{X}= & \sqrt{\operatorname{det} G}\left(\prod_{i} \delta\left(p_{i}^{(1)}+p_{i}^{(2)}\right)\right) \exp \left[-G^{i j} \sum_{n=1}^{\infty}(-1)^{n} a_{n, i}^{(1) \dagger} a_{n, j}^{(2) \dagger}\right]|0\rangle_{12}^{\prime} \\
& \times \exp \left[\frac{1}{8} \cdot 2 \alpha^{\prime} G^{i j}\left(p_{i}^{(1)}-p_{i}^{(2)}\right)\left(p_{j}^{(1)}-p_{j}^{(2)}\right)\right] \\
& \times\left(\prod_{r=1}^{2}{ }_{r}^{(0)}\left\langle p^{(r)} \mid 0\right\rangle_{r}^{(0)}\right) .
\end{aligned}
$$

Here we omitted the irrelevant constant factor multiplying the whole expression. The state $|0\rangle_{1,2}^{\prime}$ represents the vacuum with respect to the nonzero-mode oscillators, and the ground state wave function of the zero-mode oscillator in the momentum representation is

$$
{ }^{(0)}\langle p \mid 0\rangle^{(0)}=\frac{1}{(\operatorname{det} G)^{1 / 4}} \exp \left[-\frac{1}{4} \cdot 2 \alpha^{\prime} G^{i j} p_{i} p_{j}\right] \text {. }
$$

\section{A.3 $\left|V_{4}\right\rangle_{1234}$}

We obtain the solution of the continuity conditions assuming the following form:

$$
\left|V_{4}\right\rangle_{1234}^{X}=\exp \left[-\frac{1}{2} G^{i j} \sum_{r, s=1}^{4} \sum_{n, m=0}^{\infty} a_{n, i}^{(r) \dagger} V_{n m}^{r s} a_{m, j}^{(s) \dagger}\right]|0\rangle_{1234}
$$


where the matrices $V^{r s}$ are determined by the single matrix $V$ as

$$
\begin{aligned}
& V^{11}=V^{22}=V^{33}=V^{44}=\frac{1}{4}(V+\bar{V}), \\
& V^{12}=V^{23}=V^{34}=V^{41}=\frac{1}{2} C+\frac{i}{4}(V-\bar{V}), \\
& V^{13}=V^{24}=V^{31}=V^{42}=-\frac{1}{4}(V+\bar{V}), \\
& V^{14}=V^{21}=V^{32}=V^{43}=\frac{1}{2} C-\frac{i}{4}(V-\bar{V}),
\end{aligned}
$$

with $C_{n m}=(-1)^{n} \delta_{n m} . \quad V$ satisfies the following equations, which are obtained from the continuity conditions,

$$
\begin{aligned}
& (1-X) E(1+V)=(1+X) E\left(1+V^{T}\right)=0, \\
& (1-X) E^{-1}\left(1-V^{T}\right)=(1+X) E^{-1}(1-V)=0 .
\end{aligned}
$$

Here the matrices $E$ and $X$ are given by

$$
\begin{aligned}
& E_{n m}= \begin{cases}1 & (n=m=0) \\
\sqrt{2 / n} & (n=m \geq 1) \\
0 & \text { (otherwises) }\end{cases} \\
& X_{n 0}=-i \frac{\sqrt{2}}{\pi n}\left((-1)^{n}-1\right)(-1)^{(n-1) / 2} \\
& =-X_{0 n} \quad(n \geq 1) \text {, } \\
& X_{n m}=\frac{i}{\pi}(-1)^{(n-m-1) / 2}\left(1-(-1)^{n+m}\right)\left(\frac{1}{n+m}+\frac{1}{n-m}(-1)^{m}\right) \\
& =-X_{m n} \quad(n, m \geq 1, n \neq m) \text {. }
\end{aligned}
$$

All the other components of $X$ vanish. $X$ is a hermitian matrix having the properties

$$
X^{T}=\bar{X}=C X C=-X, \quad X^{2}=1 .
$$

We can see from eqs. A.14) and (A.18) that $V$ is hermitian, $V^{T}=\bar{V}=C V C$ and $V^{2}=1$. Translating to the momentum representation with respect to the zero-modes, we have

$$
\begin{aligned}
& \left(\prod_{r=1}^{4}{ }_{r}^{(0)}\left\langle p^{(r)}\right|\right)\left|V_{4}\right\rangle_{1234} \\
= & \sqrt{\operatorname{det} G} \prod_{i} \delta\left(\sum_{r=1}^{4} p_{i}^{(r)}\right) \exp G^{i j}\left[-\frac{1}{2} \sum_{r, s=1}^{4} \sum_{n, m=1}^{\infty} a_{n, i}^{(r) \dagger} V_{n m}^{\prime r s} a_{m, j}^{(s) \dagger}-\sqrt{2 \alpha^{\prime}} \sum_{r, s=1}^{4} \sum_{m=1}^{\infty} p_{i}^{(r)} V_{0 m}^{\prime r s} a_{m, j}^{(s) \dagger}\right. \\
& \left.-\alpha^{\prime} \sum_{r, s=1}^{4} p_{i}^{(r)} V_{00}^{\prime r s} p_{j}^{(s)}-\frac{\alpha^{\prime}}{8}\left(p_{i}^{(1)}-p_{i}^{(2)}+p_{i}^{(3)}-p_{i}^{(4)}\right)\left(p_{j}^{(1)}-p_{j}^{(2)}+p_{j}^{(3)}-p_{j}^{(4)}\right)\right]|0\rangle_{1234}^{\prime} \\
& \times\left(\prod_{r=1}^{4}{ }_{r}^{(0)}\left\langle p^{(r)} \mid 0\right\rangle_{r}^{(0)}\right) .
\end{aligned}
$$


Here the matrices $V^{\prime r s}$ are related to the single matrix $V^{\prime}$ by the same way as in (A.13), and $V^{\prime}$ is determined by $V$ as follows:

$$
\begin{aligned}
V_{n m}^{\prime} & =V_{n m}+\frac{V_{n 0} V_{0 m}}{1-V_{00}}, \\
V_{0 m}^{\prime} & =\frac{V_{0 m}}{1-V_{00}}, \quad V_{n 0}^{\prime}=\frac{V_{n 0}}{1-V_{00}}, \\
V_{00}^{\prime} & =\frac{V_{00}}{1-V_{00}} \quad(n, m=1,2,3, \cdots) .
\end{aligned}
$$

The explicit form of $V^{\prime}$ can be obtained by using the Neumann function method as in ref. [14]. The result is

$$
\begin{aligned}
\left(V^{\prime}+\bar{V}^{\prime}\right)_{n m} & =-2 \frac{\sqrt{n m}}{n+m}\left(u_{n} u_{m}-v_{n} v_{m}\right), \\
\left(V^{\prime}-\bar{V}^{\prime}\right)_{n m} & = \begin{cases}2 i \frac{\sqrt{n m}}{n-m}\left(u_{n} v_{m}+v_{n} u_{m}\right) & (n \neq m) \\
0 & (n=m),\end{cases} \\
\left(V^{\prime}+\bar{V}^{\prime}\right)_{0 m} & =-\frac{2}{\sqrt{m}} u_{m}, \\
\left(V^{\prime}-\bar{V}^{\prime}\right)_{0 m} & =-2 i \frac{1}{\sqrt{m}} v_{m}, \\
\left(V^{\prime}+\bar{V}^{\prime}\right)_{n 0} & =-\frac{2}{\sqrt{n}} u_{n}, \\
\left(V^{\prime}-\bar{V}^{\prime}\right)_{n 0} & =2 i \frac{1}{\sqrt{n}} v_{n}, \\
V_{00}^{\prime} & =\ln 2-\frac{1}{2},
\end{aligned}
$$

where $n$ and $m$ run over positive integers. $u_{n}$ and $v_{n}$ are given by

$$
\begin{array}{ll}
u_{n}=\left(\begin{array}{c}
-1 / 2 \\
n / 2
\end{array}\right), \quad v_{n}=0, & (n=2,4,6, \cdots), \\
u_{n}=0, \quad v_{n}=\left(\begin{array}{c}
-1 / 2 \\
(n-1) / 2
\end{array}\right), & (n=1,3,5, \cdots) .
\end{array}
$$

For the ghost sector, the result is

$$
\begin{aligned}
\left|V_{4}\right\rangle_{1234}^{g h}= & C_{+}^{2}\left(\frac{\pi}{2}\right) C_{-}^{2}\left(\frac{\pi}{2}\right) \exp \sum_{r, s=1}^{4}(-1)^{r+s}\left[\sum_{m=1}^{\infty} b_{0}^{(r)} V_{0 m}^{\prime r s} \sqrt{m} c_{-m}^{(s)}\right. \\
& \left.+\sum_{n, m=1}^{\infty} b_{-n}^{(r)} \frac{1}{\sqrt{n}} V_{n m}^{\prime r s} \sqrt{m} c_{-m}^{(s)}\right]|\Omega\rangle_{1234} .
\end{aligned}
$$


For the $Z_{4}$-Fourier transformations of $c^{(r)}$ 's:

$$
\begin{aligned}
C_{ \pm} & =\frac{1}{2}\left(i c_{ \pm}^{(1)}-c_{ \pm}^{(2)}-i c_{ \pm}^{(3)}+c_{ \pm}^{(4)}\right) \\
C_{ \pm}^{2} & =\frac{1}{2}\left(-c_{ \pm}^{(1)}+c_{ \pm}^{(2)}-c_{ \pm}^{(3)}+c_{ \pm}^{(4)}\right) \\
\bar{C}_{ \pm} & =\frac{1}{2}\left(-i c_{ \pm}^{(1)}-c_{ \pm}^{(2)}+i c_{ \pm}^{(3)}+c_{ \pm}^{(4)}\right) \\
C_{ \pm}^{4} & =\frac{1}{2}\left(c_{ \pm}^{(1)}+c_{ \pm}^{(2)}+c_{ \pm}^{(3)}+c_{ \pm}^{(4)}\right)
\end{aligned}
$$

as well as for the same combinations of $b^{(r)}$ 's, the vacuum $|\Omega\rangle_{1234}$ is given by the tensor product of the $|-\rangle$ vacuum for $\left(B^{2}, C^{2}\right)$ and the three $|+\rangle$ vacua for $\left(B^{4}, C^{4}\right),(B, C)$ and $(\bar{B}, \bar{C})$. The ghost number of the vacuum is +1 . Note that the mid-point ghost insertion $C_{+}^{2}\left(\frac{\pi}{2}\right) C_{-}^{2}\left(\frac{\pi}{2}\right)$ is needed in order for the overlap vertex to carry the correct ghost number +3 .

\section{A.4 $\left|V_{3}\right\rangle_{123}$}

We find the solution of the form

$$
\left|V_{3}\right\rangle_{123}^{X}=\exp \left[-\frac{1}{2} G^{i j} \sum_{r, s=1}^{3} \sum_{n, m=0}^{\infty} a_{n, i}^{(r) \dagger} U_{n m}^{r s} a_{m, j}^{(s) \dagger}\right]|0\rangle_{123},
$$

where the matrices $U^{r s}$ are determined by the single matrix $U$ as

$$
\begin{aligned}
U^{11}=U^{22}=U^{33} & =\frac{1}{3}(C+U+\bar{U}), \\
U^{12}=U^{23}=U^{31} & =\frac{1}{6}(2 C-U-\bar{U})+\frac{1}{6} i \sqrt{3}(U-\bar{U}), \\
U^{13}=U^{21}=U^{32} & =\frac{1}{6}(2 C-U-\bar{U})-\frac{1}{6} i \sqrt{3}(U-\bar{U}) .
\end{aligned}
$$

$U$ satisfies the following equations, which is obtained from the continuity conditions,

$$
\begin{aligned}
& (1-Y) E(1+U)=\left(1-Y^{T}\right) E\left(1+U^{T}\right)=0, \\
& \left(1+Y^{T}\right) E^{-1}\left(1-U^{T}\right)=(1+Y) E^{-1}(1-U)=0,
\end{aligned}
$$

with $Y=-\frac{1}{2} C+\frac{\sqrt{3}}{2} X$ being hermitian and satisfying $Y^{2}=1$. From eqs. (A.27) and the propertires of $Y$, we can see that $U$ is hermitian, $U^{T}=\bar{U}=C U C$ and $U^{2}=1$.

In the momentum representation with respect to the zero-modes, we have

$$
\begin{aligned}
& \left(\prod_{r=1}^{3}{ }_{r}^{(0)}\left\langle p^{(r)}\right|\right)\left|V_{3}\right\rangle_{123} \\
= & \sqrt{\operatorname{det} G} \prod_{i} \delta\left(\sum_{r=1}^{3} p_{i}^{(r)}\right) \exp G^{i j}\left[-\frac{1}{2} \sum_{r, s=1}^{3} \sum_{n, m=1}^{\infty} a_{n, i}^{(r) \dagger} U_{n m}^{\prime r s} a_{m, j}^{(s) \dagger}\right.
\end{aligned}
$$




$$
\begin{aligned}
& \left.-\sqrt{2 \alpha^{\prime}} \sum_{r, s=1}^{3} \sum_{m=1}^{\infty} p_{i}^{(r)} U_{0 m}^{\prime r s} a_{m, j}^{(s) \dagger}-\frac{1}{2} \cdot 2 \alpha^{\prime} \sum_{r, s=1}^{3} p_{i}^{(r)} U_{00}^{\prime r s} p_{j}^{(s)}\right]|0\rangle_{123}^{\prime} \\
& \times\left(\prod_{r=1}^{3}{ }_{r}^{(0)}\left\langle p^{(0)} \mid 0\right\rangle_{r}^{(0)}\right) .
\end{aligned}
$$

The matrices $U^{\prime r s}$ 's are also written by the single matrix $U^{\prime}$. Their relation is in the same form as in (A.26). Also $U^{\prime}$ is determined by $U$ as

$$
\begin{aligned}
& U_{n m}^{\prime}=U_{n m}+\frac{U_{n 0} U_{0 m}}{1-U_{00}}, \\
& U_{0 m}^{\prime}=\frac{U_{0 m}}{1-U_{00}}, \quad U_{n 0}^{\prime}=\frac{U_{n 0}}{1-U_{00}}, \\
& U_{00}^{\prime}=\frac{U_{00}}{1-U_{00}} \quad(n, m=1,2,3, \cdots) .
\end{aligned}
$$

The explicit form of $U^{\prime}$ is presented as follows:

For $n \neq m, n, m \geq 1$,

$$
\begin{aligned}
\left(U^{\prime}+\bar{U}^{\prime}\right)_{n m} & = \begin{cases}(-1)^{n+1} \sqrt{n m}\left(\frac{A_{n} B_{m}+B_{n} A_{m}}{n+m}+\frac{A_{n} B_{m}-B_{n} A_{m}}{n-m}\right) & (n+m: \text { even }) \\
0 & (n+m: \text { odd }),\end{cases} \\
\left(U^{\prime}-\bar{U}^{\prime}\right)_{n m} & = \begin{cases}0 & (n+m: \text { even }) \\
i \sqrt{n m}\left(\frac{A_{n} B_{m}-B_{n} A_{m}}{n+m}+\frac{A_{n} B_{m}+B_{n} A_{m}}{n-m}\right) & (n+m: \text { odd }),\end{cases} \\
\left(U^{\prime}+\bar{U}^{\prime}\right)_{0 m} & = \begin{cases}-\frac{2}{\sqrt{m}} A_{m} & (m: \text { even }) \\
0 & (m: \text { odd }),\end{cases} \\
\left(U^{\prime}-\bar{U}^{\prime}\right)_{0 m} & = \begin{cases}0 & (m: \text { even }) \\
-i \frac{2}{\sqrt{m}} A_{m} & (n: \text { oven })\end{cases} \\
\left(U^{\prime}+\bar{U}^{\prime}\right)_{n 0} & = \begin{cases}-\frac{2}{\sqrt{n}} A_{n} & (n: \text { odd }), \\
0 & (n: \text { even }) \\
0 & (n: \text { odd }) . \\
i \frac{2}{\sqrt{n}} A_{n} & \end{cases}
\end{aligned}
$$

For the diagonal elements,

$$
\begin{aligned}
U_{00} & =\frac{1}{2} \ln \left(3^{3} / 2^{4}\right)-\frac{1}{2}, \\
\left(U^{\prime}+\bar{U}^{\prime}\right)_{n n} & =(-1)^{n+1}\left(A_{n} B_{n}+1\right)-n \Delta_{n}, \\
\left(U^{\prime}-\bar{U}^{\prime}\right)_{n n} & =0 \quad(n \geq 1) .
\end{aligned}
$$

Here $A_{n}$ and $B_{n}$ are given by

$$
\begin{aligned}
& A_{n}= \begin{cases}(-1)^{n / 2} a_{n} & (n: \text { even }) \\
(-1)^{(n-1) / 2} a_{n} & (n: \text { odd }),\end{cases} \\
& B_{n}= \begin{cases}(-1)^{n / 2} b_{n} & (n: \text { even }) \\
(-1)^{(n-1) / 2} b_{n} & (n: \text { odd }),\end{cases}
\end{aligned}
$$




$$
\left(\frac{1+x}{1-x}\right)^{1 / 3}=\sum_{n=0}^{\infty} a_{n} x^{n}, \quad\left(\frac{1+x}{1-x}\right)^{2 / 3}=\sum_{n=0}^{\infty} b_{n} x^{n}
$$

Also $\Delta_{n}$ 's are

$$
\Delta_{n}= \begin{cases}-\frac{\sqrt{3}}{\pi}(-1)^{n / 2}\left(\tilde{O}_{n}^{a} B_{n}-\tilde{O}_{n}^{b} A_{n}\right) & (n=2,4,6, \cdots) \\ \frac{\sqrt{3}}{\pi}(-1)^{(n-1) / 2}\left(\tilde{E}_{n}^{a} B_{n}-\tilde{E}_{n}^{b} A_{n}\right) & (n=1,3,5, \cdots),\end{cases}
$$

where $\tilde{O}_{n}^{a}, \tilde{E}_{n}^{a}, \tilde{O}_{n}^{b}$ and $\tilde{E}_{n}^{b}$ can be written in terms of $a_{n}$ 's and $b_{n}$ 's as

$$
\begin{aligned}
& \tilde{O}_{0}^{a}=\frac{\pi \sqrt{3}}{4} \ln \frac{3^{3}}{2^{4}}, \quad \tilde{E}_{1}^{a}=\frac{\pi}{\sqrt{3}}\left(\ln \frac{3}{2}+\frac{1}{6}\right), \\
& \tilde{E}_{n}^{a}=\frac{\pi \sqrt{3}}{2}\left[\left(\ln \frac{3}{2}+\frac{1}{6}\right) a_{n}-a_{n+1}\right. \\
& \left.+\sum_{l=0}^{n-1} \frac{(-1)^{l}}{l+1} a_{n-l-1}\left(a_{l+2}+\frac{1}{2} a_{l}\right)\right] \quad(n=3,5,7, \cdots), \\
& \tilde{O}_{n}^{a}=\frac{\pi \sqrt{3}}{2}\left[\left(\ln \frac{3}{2}+\frac{1}{6}\right) a_{n}-a_{n+1}\right. \\
& \left.+\sum_{l=0}^{n-1} \frac{(-1)^{l}}{l+1} a_{n-l-1}\left(a_{l+2}+\frac{1}{2} a_{l}\right)\right] \quad(n=2,4,6, \cdots), \\
& \tilde{O}_{0}^{b}=\frac{\pi \sqrt{3}}{4} \ln 3, \quad \tilde{E}_{1}^{b}=\frac{2 \pi}{\sqrt{3}}\left(\ln \frac{3}{2}-\frac{1}{12}\right), \\
& \tilde{E}_{n}^{b}=\frac{\pi \sqrt{3}}{4}\left[\left(2 \ln \frac{3}{2}-\frac{1}{6}\right) b_{n}-b_{n+1}\right. \\
& \left.+\sum_{l=0}^{n-1} \frac{(-1)^{l}}{l+1} b_{n-l-1}\left(a_{l+2}-\frac{1}{2} b_{l}\right)\right] \quad(n=3,5,7, \cdots), \\
& \tilde{O}_{n}^{b}=\frac{\pi \sqrt{3}}{4}\left[\left(2 \ln \frac{3}{2}-\frac{1}{6}\right) b_{n}-b_{n+1}\right. \\
& \left.+\sum_{l=0}^{n-1} \frac{(-1)^{l}}{l+1} b_{n-l-1}\left(a_{l+2}-\frac{1}{2} b_{l}\right)\right] \quad(n=2,4,6, \cdots) .
\end{aligned}
$$

Finally we show the result of the ghost 3-string overlap vertex:

$$
\left|V_{3}\right\rangle_{123}^{g h}=\exp \sum_{r, s=1}^{3}\left[\sum_{n, m=1}^{\infty} b_{-n}^{(r)} \frac{1}{\sqrt{n}} W_{n m}^{r s} \sqrt{m} c_{-m}^{(s)}+\sum_{m=1}^{\infty} b_{0}^{(r)} W_{0 m}^{r s} \sqrt{\frac{m}{2}} c_{-m}^{(s)}\right]|\Omega\rangle_{123},
$$

where the matrices $W^{r s}$ 's are related to the single matrix $W$ as

$$
\begin{aligned}
& W^{11}=W^{22}=W^{33}=\frac{1}{3}(-C+W+\bar{W}), \\
& W^{12}=W^{23}=W^{31}=\frac{1}{6}(-2 C-W-\bar{W})+i \frac{\sqrt{3}}{6}(W-\bar{W}),
\end{aligned}
$$




$$
W^{13}=W^{21}=W^{32}=\frac{1}{6}(-2 C-W-\bar{W})-i \frac{\sqrt{3}}{6}(W-\bar{W}),
$$

which is different from the relation for $U$ (A.26) by the sign in front of $C$. The explicit from of $W$ is given as follows:

For $n, m \geq 1$ and $n \neq m$,

$$
\begin{aligned}
& (W+\bar{W})_{n m}= \begin{cases}(-1)^{n+1} \sqrt{n m}\left(\frac{A_{n} B_{m}+B_{n} A_{m}}{n+m}-\frac{A_{n} B_{m}-B_{n} A_{m}}{n-m}\right) & (n+m: \text { even }) \\
0 & (n+m: \text { odd }),\end{cases} \\
& (W-\bar{W})_{n m}= \begin{cases}0 & (n+m: \text { even }) \\
i \sqrt{n m}\left(\frac{A_{n} B_{m}-B_{n} A_{m}}{n+m}-\frac{A_{n} B_{m}+B_{n} A_{m}}{n-m}\right) & (n+m: \text { odd }),\end{cases} \\
& (W+\bar{W})_{0 m}= \begin{cases}-2 \sqrt{\frac{2}{m}} B_{m} & (m: \text { even }) \\
0 & (m: \text { odd }),\end{cases} \\
& (W-\bar{W})_{0 m}= \begin{cases}0 & (m: \text { oven }) \\
i 2 \sqrt{\frac{2}{m}} B_{m} & \end{cases} \\
& W_{n 0}=\bar{W}_{n 0}=0 .
\end{aligned}
$$

For the diagonal elements,

$$
\begin{aligned}
& W_{00}=\bar{W}_{00}=0 \\
& (W+\bar{W})_{n n}=(-1)^{n}\left(1-a_{n} b_{n}\right)+n \Delta_{n}, \\
& (W-\bar{W})_{n n}=0 \quad(n \geq 1) .
\end{aligned}
$$

The vacuum $|\Omega\rangle_{123}$ is the tensor product of the three vacua $|+\rangle_{r}(r=1,2,3)$, whose ghost number is $+\frac{3}{2}$. This is the correct ghost number which the three-string overlap vertex should have. 


\section{References}

[1] E. Witten, Non-Commutative Geometry and String Field Theory, Nucl. Phys. B268 (1986) 253.

[2] E. Witten, Interacting Field Theory of Open Superstrings, Nucl. Phys. B276 (1986) 291.

[3] J. Polchinski, Dirichlet-branes and Ramond-Ramond charges, Phys. Rev. Lett. 75 (1995) 4724, hep-th/9510017.

[4] E. Witten, Bound States of Strings and p-Branes, Nucl. Phys. B460 (1996) 335, hep-th/9510135.

[5] A. Abouelsaood, C. G. Callan, C. R. Nappi and S. A. Yost, Open Strings in Background Gauge Fields, Nucl. Phys. B280 (1987) 599.

[6] Chong-Sun Chu and Pei-Ming Ho, Noncommutative Open String and D-brane, Nucl. Phys. B550 (1999) 151, hep-th/9812219; Constrained Quantization of Open String in Background B Field and Noncommutative D-brane, hep-th/9906192.

[7] F. Ardalan, H. Arfraei, and M. M. Sheikh-Jabbari, Noncommutative Geometry From Strings and Branes, JHEP 9902 (1999) 016, hep-th/9810072; Dirac Quantization of Open Strings and Noncommutativity in Branes, hep-th/9906161.

M. M. Sheikh-Jabbari, Open Strings in a B-Field Background as Electoric Dipoles, Phys. Lett. B455 (1999) 129, hep-th/9901080.

V. Schomerus, D-branes and Deformation Quantization, JHEP 9906 (1999) 030, hep-th/9903205.

[8] N. Ishibashi, p-Branes from ( $p-2)$-Branes in the Bosonic String Theory, Nucl. Phys. B539 (1999) 107, hep-th/9804163.

[9] M. Kato and T. Kuroki, World Volume Noncommutativity Versus Target Space Noncommutativity, JHEP 03 (1999) 012, hep-th/9902004.

[10] N. Seiberg and E. Witten, String Theory and Noncommutative Geometry, JHEP 9909 (1999) 032, hep-th/9908142.

[11] Y. Okawa, Derivative Corrections to Dirac-Born-Infeld Lagrangian and Noncommutative Gauge Theory, hep-th/9909132.

T. Asakawa and I. Kishimoto, Comments on Gauge Equivalence in Noncommutative Geometry, JHEP 9911 (1999) 024, hep-th/9909139.

A. Fayyazuddin and M. Zabzine, A Note on Bosonic Open Strings in Constant B Field, hep-th/9911018.

M. M. Sheikh-Jabbari, On the Deformation of $\Lambda$-Symmetry in B-field Background, 
hep-th/9910258.

T. Lee, Noncommutative Dirac-Born-Infeld Action for D-brane, hep-th/9912038.

O. Andreev and H. Dorn, On Open String $\sigma$-Model and Noncommutative Gauge Fields, hep-th/9912070.

M. Kreuzer and J. Zhou, $\Lambda$-Symmetry and Background Independence of Noncommutative Gauge Theory on $\mathbf{R}^{n}$, hep-th/9912174.

[12] N. Ishibashi, A Relation between Commutative and Noncommutative Descriptions of D-branes, hep-th/9909176.

[13] K. Okuyama, A Path Integral Representation of the Map between Commutative and Noncommutative Gauge Fields, hep-th/9910138.

[14] D. J. Gross and A. Jevicki, Operator Formulation of Interacting String Field Theory (I), Nucl. Phys. B283 (1987) 1.

[15] D. J. Gross and A. Jevicki, Operator Formulation of Interacting String Field Theory (II), Nucl. Phys. B287 (1987) 225.

[16] D. J. Gross and A. Jevicki, Operator Formulation of Interacting String Field Theory (III) NSR superstring, Nucl. Phys. B293 (1987) 29.

[17] T. Kugo and B. Zwiebach, Target Space Duality as a Symmetry of String Field Theory, Prog. Theo. Phys. 87 (1982) 801.

[18] T. Yoneya, in Seventh Workshop on Grand Unification /ICOBAN'86, eds. J. Arafune (World Scientific, 1986).

H. Hata, K. Itoh, T. Kugo, H. Kunitomo and K. Ogawa, Pregeometrical String Field Theory: Creation of Space-Time and Motion, Phys. Lett. 175B (1986) 138.

[19] G. T. Horowitz, J. Lykken, R. Rohm and A. Strominger, Purely Cubic Action for String Field Theory, Phys. Rev. Lett. 57 (1986) 283.

[20] R. Dearnaley, Adding Chan-Paton Factors to Witten's String Field Theory, Class. Quantum. Grav. 6 (1989) 1203.

[21] R. Dearnaley, The Zero-Slope Limit of Witten's String Field Theory with Chan-Paton Factors, Nucl. Phys. B334 (1990) 217.

[22] E. Cremmer, A. Schwimmer and C. Thorn, The Vertex Function in Witten's Formulation of String Field Theory, Phys. Lett. B179 (1986) 57.

[23] T. Banks, W. Fischler, S. Shenker and L. Susskind, M theory as a Matrix Model: $a$ Conjecture, Phys. Rev. D55 (1997) 5112, hep-th/9610043.

[24] T. Banks, N. Seiberg and S. Shenker, Branes from Matrices, Nucl. Phys. B490 (1997) 91, hep-th/9612157. 
[25] N. Ishibashi, H. Kawai, Y. Kitazawa and A. Tsuchiya, A Large-N Reduced Model as Superstring, Nucl. Phys. B498 (1997) 467, hep-th/9612115.

[26] H. Aoki, N. Ishibashi, S. Iso, H. Kawai, Y. Kitazawa and T. Tada, Noncommutative Yang-Mills in IIB Matrix Model, hep-th/9908141. 